\title{
The transmission spectrum of Earth-size transiting planets
}

\author{
D. Ehrenreich ${ }^{1}$, G. Tinetti ${ }^{2}$, A. Lecavelier des Etangs ${ }^{1}$, A. Vidal-Madjar ${ }^{1}$, and F. Selsis ${ }^{3}$ \\ 1 Institut d'Astrophysique de Paris, CNRS (UMR 7095) - Université Pierre \& Marie Curie, 98bis boulevard Arago, 75014 Paris, France \\ e-mail: ehrenreich@iap.fr \\ 2 NASA Astrobiology Institute, California Institute of Technology, IPAC, MS 220-6, 1200 E. California, Pasadena, 91125 (CA), USA \\ 3 Centre de Recherche Astronomique de Lyon, École Normale Supérieure, 47 allée d'Italie, 69364 Lyon Cedex 7, France
}

Received 19 July 2005 / Accepted 29 September 2005

\section{ABSTRACT}

\begin{abstract}
A variety of terrestrial planets with different physical parameters and exotic atmospheres might plausibly exist outside our Solar System, waiting to be detected by the next generation of space-exploration missions. Some of these planets might be transiting their parent star. We present here a detailed study of the atmospheric signatures of transiting Earth-size exoplanets. We focus on a limited number of significant examples, for which we discuss the detectability of some of the possible molecules present in their atmospheres, such as water $\left(\mathrm{H}_{2} \mathrm{O}\right)$, carbon dioxide $\left(\mathrm{CO}_{2}\right)$, ozone $\left(\mathrm{O}_{3}\right)$, or molecular oxygen $\left(\mathrm{O}_{2}\right)$. To this purpose, we developed a model to simulate transmission spectra of Earth-size exoplanets from the ultraviolet (UV) to the near infrared (NIR). According to our calculations, the signatures of planetary atmospheres represent an absorption of a few parts-per-million (ppm) in the stellar flux. The atmospheres of a few Earth-like planets can be detected with a 30-40 m telescope. The detection of the extensive atmospheres of tens of small satellites of giant exoplanets and hundreds of hypothetical ocean-planets can be achieved with 20-30 m and 10-20 m instruments, respectively, provided all these planets are frequent and they are efficiently surveyed. We also found that planets around $\mathrm{K}$ stars are favored, mainly because these stars are more numerous and smaller compared to $\mathrm{G}$ or $\mathrm{F}$ stars. While not addressed in this study, limitations might come from stellar photometric micro-variability.
\end{abstract}

Key words. planets and satellites: general

\section{Introduction}

The Earth is the only known example of a life-hosting world, even though terrestrial exoplanets have been sought since the discovery of the first Earth-mass exoplanets by Wolszczan \& Frail (1992). However, planets similar to the Earth, Venus, or Mars in size, density, or orbital parameters are still beyond the reach of our present capability to detect planets around normal stars.

Until now, mostly giant exoplanets have been discovered. Remarkable progress has been made recently with the discovery of planets in the mass range of 14 to 21 Earth masses $\left(M_{\oplus}\right.$, see McArthur et al. 2004; Santos et al. 2004), and more recently a $\sim 7.5 M_{\oplus}$ planet orbiting GJ 876 (Rivera et al. 2005). We may speculate, then, that smaller planets with sizes down to that of the Earth might be observed in the near future. Among the 161 planets $^{1}$ detected so far, eight have been discovered or re-discovered as they were transiting their parent star, producing a photometric occultation. The last transiting planet identified is a Saturn-mass planet orbiting HD 149026, a bright

\footnotetext{
${ }^{1}$ From J. Schneider's Extrasolar Planets Encyclopædia at vo.obspm.fr/exoplanetes/encyclo/encycl.html. See also the web page of the IAU Working Group on Extrasolar Planets at www. ciw. edu/boss/IAU/div3/wgesp.
}

$V=8.15$ G0 IV star (Sato et al. 2005). The first transiting giant exoplanet, HD 209458b to be discovered (Henry et al. 2000; Charbonneau et al. 2000; Mazeh et al. 2000) is the object of intense investigations dedicated to characterizing its hot atmosphere.

Probing planetary atmospheres by stellar occultations is an effective method used for a lot of planets and their satellites in the Solar System, from Venus to Charon (see, e.g., Elliot \& Olkin 1996). With this technique, we can observe the thin atmospheric ring surrounding the optically thick disk of the planet: the limb. In the case of giant exoplanets, though, the star is only partially occulted (1.6\% for the transiting planet HD 209458b). The spectrum of the starlight transmitted and filtered by the lower and thick giant exoplanet atmosphere consequently presents extremely weak absorption features (from $10^{-3}$ to $10^{-4}$, see Seager \& Sasselov 2000; Hubbard et al. 2001; Brown 2001).

Despite the difficulties, these dim signatures were able to be detected: Charbonneau et al. (2002) measured the lower atmosphere of HD 209458b when they detected a $(2.32 \pm 0.57) \times 10^{-4}$ photometric diminution in the sodium doublet line of the parent star at $589.3 \mathrm{~nm}$. However, its upper atmosphere, which extends up to several planet radii, shows even larger signatures. Vidal-Madjar et al. (2003, 2004) 
observed a $15 \pm 4 \%$ absorption in the Lyman $\alpha\left(\mathrm{Ly}_{\alpha}\right)$ emission line of HD 209458 at $121.57 \mathrm{~nm}$, as well as absorptions from atomic carbon $(7.5 \pm 3.5 \%)$ and oxygen $(13 \pm 4.5 \%)$ in the upper atmosphere. In this work, we will discuss the possibility of detecting and to characterizing the lower atmospheres of exoplanets using signatures comparable in origin to the one detected by Charbonneau et al. (2002).

The idea is to extend the use of transmission spectroscopy to hypothetical Earth-size planets (Schneider 1994; Webb \& Wormleaton 2001). We estimate that these exoplanets present at least two orders of magnitude lower signal than gaseous giants, as the transit of the planet itself would have a dimming of $\sim 10^{-5}$. The transit depth, $\Delta F / F$ where $F$ is the stellar flux, can be expressed as $\left(R_{\mathrm{P}} / R_{\star}\right)^{2}$, with $R_{\mathrm{P}}$ and $R_{\star}$ standing for the radii of the planet and the star, respectively. The atmospheres of Earth-size exoplanets should span over more than $\sim 100$-km height without considering potential upper atmospheres. Depending on their transparency - which would give an equivalent optically thick layer of $\sim 10 \mathrm{~km}-$ the expected occultations caused by atmospheric absorptions should be $\sim 10^{-7}$ to $\sim 10^{-6}$.

Earth-size planets are probably the most challenging objects to detect with transmission spectroscopy. The orders of magnitude given above, in fact, raise many questions: is it realistic to seek possible features that dim, and do so with an instrumentation that might or might not be available in the near future? What are the strongest signatures we should expect? What kind of planet could be the best candidate to look at?

We have developed a one-dimensional model of transmission at the limb to give quantitative answers to these questions. Since we use stellar light to explore the planetary atmospheres, we chose to focus on the wavelength range where the largest number of photons is available, i.e., between 200 and $2000 \mathrm{~nm}$. This model is described in Sect. 2. The detectability of the selected atmospheres depends on the signal-to-noise ratio $(S / N)$ that is achievable with a space telescope spectrograph. The constraints on idealized observations and the method we used to calculate their $S / N$ are described in Sect. 3. Finally, results for the specified cases are given and discussed in Sect. 4.

\section{Model description}

\subsection{Geometric description of the model}

The general geometry of a transiting system is described by Brown (2001). In the present work we consider a non-transient occultation for the "in transit" phase, with a null phase configuration (configuration 2 in Brown's Fig. 1), that is, the planet is centered in the line of sight with respect to the star. This configuration both maximizes the area of the atmosphere that is filtering the stellar light and minimizes any effects linked to the stellar limb darkening (Seager \& Sasselov 2000).

The stellar light is filtered through the atmospheric limb of the planet, as sketched in Fig. 1. In the following we explain the integration of the atmospheric opacity in detail along a stellar light path (or cord) through the limb of the planet.

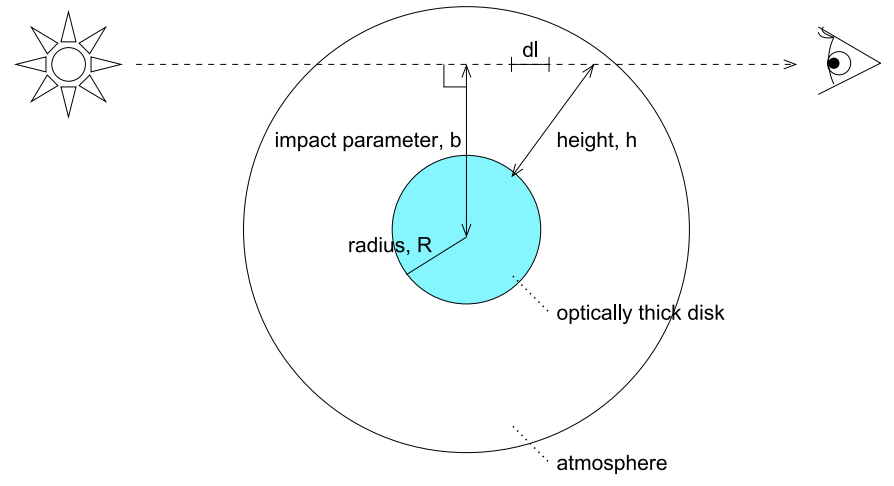

Fig. 1. Sketch of the transmission of the stellar light through the planetary limb. The planet itself, i.e. the "solid" disk (in grey) is optically thick at all wavelengths. The quantity $\mathrm{d} l$ is the elemental length along the line of sight. In the calculation, we prefer to use height $h$ instead of $l$. The scale in the figure has been distorted for clarity.

\subsubsection{Opacity along the line of sight}

We calculate the total opacity $\tau_{\lambda}$ of the model atmosphere along a cord, parallel to the line of sight, as the sum of the opacity of each species $i$ present in the atmosphere, $\tau_{\lambda}=\sum_{i} \tau_{\lambda, i}$. We can calculate the opacity along the cord as a function of its impact parameter $b$

$\tau_{\lambda, i}(b)=2 \int_{0}^{+\infty} A_{\lambda, i} \rho_{i}(h) \mathrm{d} l$,

where $A_{\lambda, i}$ is the absorption coefficient for species $i$ at wavelength $\lambda$, expressed in $\mathrm{cm}^{2} \mathrm{~g}^{-1}$, and $\rho_{i}(h)$ is the mass density in $\mathrm{g} \mathrm{cm}^{-3}$ of the species $i$ at an altitude $h$ in the atmosphere.

Now, re-expressing Eq. (1) as a function of the height $z=$ $h+R_{\mathrm{P}}$, with $R_{\mathrm{P}}$ the planet radius, we obtain:

$\tau_{\lambda, i}(b)=2 \int_{b}^{b_{\max }} A_{\lambda, i} \rho_{i}\left(z-R_{\mathrm{P}}\right) \frac{z \mathrm{~d} z}{\sqrt{z^{2}-b^{2}}}$

where $b_{\max }$ is the height of the higher atmospheric level we are considering. The method to estimate $b_{\max }$ is presented in Sect. 2.2.3.

\subsubsection{Spectrum ratio}

Consider the stellar flux received by the observer during the planetary transit to be $F_{\text {in }}$, and the flux received when the planet is not occulting the star to be $F_{\text {out }}$. Brown (2001) defined $\mathfrak{R}$ as the ratio between those two quantities, and $\mathfrak{R}^{\prime}$ (the so-called spectrum ratio) as $\mathfrak{R}^{\prime}=\mathfrak{R}-1$. Here, $\mathfrak{R}^{\prime}$ is the sum of two distinct types of occultations:

- The occultation by the "solid" surface of the planet, optically thick at all wavelengths. Projected along the line of sight, this is a disk of radius $R_{\mathrm{P}}$, and the occultation depth is simply $\left(R_{\mathrm{P}} / R_{\star}\right)^{2}$.

- The wavelength-dependent occultation by the thin ring of gaseous components that surrounds the planetary disk, which can be expressed as $\Sigma_{\lambda} /\left(\pi R_{\star}^{2}\right)$. The area, $\Sigma_{\lambda}$, is the 
atmospheric equivalent surface of absorption and may be calculated as:

$$
\Sigma_{\lambda}=\int_{R_{\mathrm{P}}}^{b_{\max }} 2 \pi b \mathrm{~d} b\left[1-\mathrm{e}^{-\tau_{\lambda}(b)}\right] .
$$

The resulting spectrum ratio is:

$$
\mathfrak{R}^{\prime}(\lambda)=-\frac{\Sigma_{\lambda}+\pi R_{\mathrm{P}}^{2}}{\pi R_{\star}^{2}} .
$$

Note that $\mathfrak{R}^{\prime}<0$.

\subsection{Description of the atmospheric profiles}

Along a single cord, stellar photons cross several levels of the spherically stratified atmosphere. We generate an atmospheric model using the vertical profiles from Tinetti et al. (2005a,b) and Fishbein et al. (2003) for the Earth and from the Venus International Reference Atmosphere (VIRA, Kliore et al. 1985) for Venus. These atmospheric data include the profiles of pressure, $p$, temperature, $T$, and various mixing ratios, $Y$. The atmospheres are initially sampled in 50 levels, ranging from the ground level to an altitude of about $80 \mathrm{~km}$ for the Earth and about $50 \mathrm{~km}$ for Venus. Both profiles stop below the homopause, so we assume hydrostatic equilibrium for the vertical pressure gradient.

A useful quantity to describe atmospheres in hydrostatic equilibrium is the scale height, $H$, i.e. the height above which the pressure decreases by a factor $e$. The scale height depends explicitly on the temperature as $H=k \mathcal{N}_{\mathrm{A}} T /(\mu g)$, where $k$ and $\mathcal{N}_{\mathrm{A}}$ are the Boltzmann's and Avogadro's constants, while $\mu$ is the mean molar mass of the atmospheric gas. Since $g$ is the acceleration due to gravity, $H$ also implicitly depends on the radius and the density of the planet ${ }^{2}$. Consequently, less dense objects are likely to have more extensive atmospheres, so they are easier to detect (Brown 2001).

Density and the size of planets are therefore key parameters for this work. In order to estimate their influence, we tested a set of different planetary types ranging from the Titan-like giant planet's satellite $\left(\rho_{\mathrm{P}} \approx 2 \mathrm{~g} \mathrm{~cm}^{-3}, R_{\mathrm{P}} \approx 0.5\right.$ Earth radius $0.5 R_{\oplus}$ ) to the "super-Earth" object ( $\rho_{\mathrm{P}} \approx 6 \mathrm{~g} \mathrm{~cm}^{-3}, R_{\mathrm{P}} \approx 2 R_{\oplus}$ ). For the physical properties of plausible, theoretically predicted planets such as a "super-Earth", we used the mass-radius relation model from Dubois (2004) and from Sotin et al. (2005). Our atmospheric model allowed the vertical profiles to be rescaled depending on the acceleration due to the gravity of the planet and the atmospheric pressure at the reference level.

\subsubsection{Molecular composition of the atmosphere}

Our simplified atmospheric profiles contain only the species that may produce interesting spectral signatures in the chosen wavelength range $(0.2$ to $2 \mu \mathrm{m})$, viz. water vapor $\left(\mathrm{H}_{2} \mathrm{O}\right)$, carbon dioxide $\left(\mathrm{CO}_{2}\right)$, ozone $\left(\mathrm{O}_{3}\right)$, and molecular oxygen $\left(\mathrm{O}_{2}\right)$. Molecular nitrogen $\left(\mathrm{N}_{2}\right)$ has also been considered, though lacking marked electronic transitions from the UV to the near IR.

\footnotetext{
2 To avoid confusion between the density of the atmosphere and the mean density of the planet, the latter is denoted $\rho_{\mathrm{P}}$.
}

Nevertheless, it is a major species in Earth's atmosphere, and it has a detectable signature via Rayleigh scattering at short wavelengths.

We considered three types of atmospheres: (A) $\mathrm{N}_{2} / \mathrm{O}_{2}$ rich, (B) $\mathrm{CO}_{2}$-rich, and (C) $\mathrm{N}_{2} / \mathrm{H}_{2} \mathrm{O}$-rich cases. The first two types can be associated with existing planetary atmospheres, Earth and Venus. The last type (C) could correspond to the atmosphere of an Earth-mass volatile-rich planet, such as the "ocean-planet" described by Léger et al. (2004). The bases for building a "toy model" of an $\mathrm{H}_{2} \mathrm{O}$-rich atmosphere are found in Léger et al. (2004) and Ehrenreich et al. (2005b, see Sect. 2.2.5)

The vertical gradients in the chemical composition and temperature of each of these atmospheres are plotted in Fig. 2 $\left(\mathrm{N}_{2} / \mathrm{O}_{2}\right.$-rich), Fig. $3\left(\mathrm{CO}_{2}\right.$-rich $)$, and Fig. $4\left(\mathrm{~N}_{2} / \mathrm{H}_{2} \mathrm{O}\right.$-rich $)$. Table 1 summarizes the mean chemical compositions of these model atmospheres.

\subsubsection{Temperature profiles}

As mentioned above, we used the vertical temperature profiles of Earth and Venus as prototypes for $\mathrm{N}_{2} / \mathrm{O}_{2}$-rich and $\mathrm{CO}_{2}$-rich atmospheres (see Sect. 2.4). Moreover, we assumed an isothermal profile in the thermosphere, instead of the real one. This is an arbitrary but conservative choice, since the temperature should instead rise in the thermosphere enhancing the atmosphere's detectability (see Sect. 4.2.2).

\subsubsection{Upper limit of the atmosphere}

We set the profiles to extend up to a critical height $b_{\max }$ from the center of the planet, or $h_{\max }$ from the surface $\left(b_{\max }=R_{\mathrm{P}}+h_{\max }\right)$. This limit corresponds to the altitude above which the molecular species we considered $\left(\mathrm{H}_{2} \mathrm{O}, \mathrm{O}_{3}\right.$, $\mathrm{CO}_{2}, \mathrm{O}_{2}$ ) are likely to be destroyed or modified either by photodissociating or ionizing radiations, such as $\mathrm{Ly}_{\alpha}$ or extreme-UV (EUV).

As a result, the critical height corresponds to the mesopause on Earth (at $\approx 85 \mathrm{~km}$ ). The column density of the terrestrial atmosphere above that altitude, $\mathcal{N}_{\geq 85 \mathrm{~km}}$, is sufficient to absorb all $\mathrm{Ly}_{\alpha}$ flux. In fact, as the number density of the atmospheric gas, $n(h)$, decreases exponentially with height, we can simply consider $\mathcal{N}_{\geq 85 \mathrm{~km}} \propto n_{85 \mathrm{~km}} \cdot H_{85} \mathrm{~km}$, where $n_{85} \mathrm{~km}$ and $H_{85} \mathrm{~km}$ are the density and the scale height of the terrestrial atmosphere at $85 \mathrm{~km}$, respectively.

Similarly, we set upper limit $h_{\max }$ of a given atmosphere to the altitude below which the photo-dissociating photons are absorbed. We assumed that $h_{\max }$ is the altitude where the column density equals that of the terrestrial atmosphere at $85 \mathrm{~km}$, i.e. $n_{h_{\max }} \cdot H_{h_{\max }}=\left(n_{85 \mathrm{~km}}\right)_{\oplus} \cdot\left(H_{85} \mathrm{~km}\right)_{\oplus}$. We determined $h_{\max }$ by scaling this equation.

Values of $h_{\max }$ for the different models are given in Table 2. As for neutral elements absorbing light below $h_{\max }$, it is likely that ionized elements are absorbing light above this limit, though we do not include this effect in the model. 
Table 1. Mean volume mixing ratio of atmospheric absorbers for the different types of model atmospheres considered. $(*)$ Ozone is only present in model A1.

\begin{tabular}{cccccccc}
\hline \hline Type & $\mu\left(\mathrm{g} \mathrm{mol}^{-1}\right)$ & $Y_{\mathrm{N}_{2}}(\%)$ & $Y_{\mathrm{H}_{2} \mathrm{O}}(\%)$ & $Y_{\mathrm{CO}_{2}}(\%)$ & $Y_{\mathrm{O}_{2}}(\%)$ & $Y_{\mathrm{O}_{3}}(\%)$ & Used for models \\
\hline $\mathrm{N}_{2} / \mathrm{O}_{2}$-rich & 28.8 & 78 & 0.3 & 0.03 & 21 & $<10^{-3}$ & $\mathrm{~A} 1, \mathrm{~A} 2, \mathrm{~A} 3$ \\
$\mathrm{CO}_{2}$-rich & 43.3 & 4 & $3 \times 10^{-4}$ & 95 & 0 & 0 & $\mathrm{~B} 1, \mathrm{~B} 2, \mathrm{~B} 3$ \\
$\mathrm{~N}_{2} / \mathrm{H}_{2} \mathrm{O}$-rich & 28.7 & 80 & 10 & 10 & 0 & 0 & $\mathrm{C} 1, \mathrm{C} 2, \mathrm{C} 3$ \\
\hline
\end{tabular}

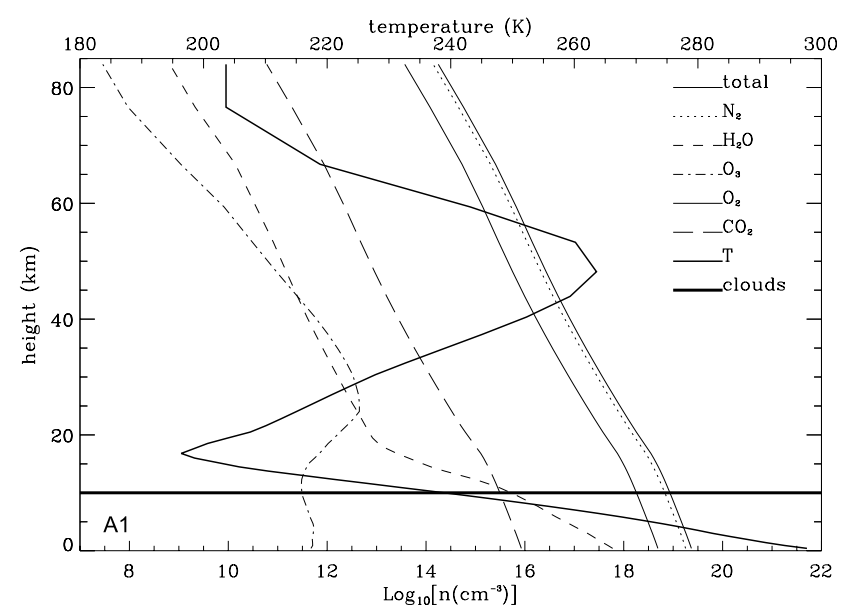

Fig. 2. Atmospheric profiles, A1. The plot shows the total number density profile (thin solid line) of the atmosphere in $\mathrm{cm}^{-3}$ and those of the five species included in our model, namely, $\mathrm{N}_{2}$ (dotted line), $\mathrm{O}_{2}$ (dashdot-dot-dotted line), $\mathrm{H}_{2} \mathrm{O}$ (dashed line), $\mathrm{CO}_{2}$ (long-dashed line), and $\mathrm{O}_{3}$ (dash-dotted line). Temperature (thick line up to $80 \mathrm{~km}$ ) and mixing ratios of the different species are those of Earth. Temperature is assumed to be constant above that height. The thickest horizontal line shows the position of the cloud layer.

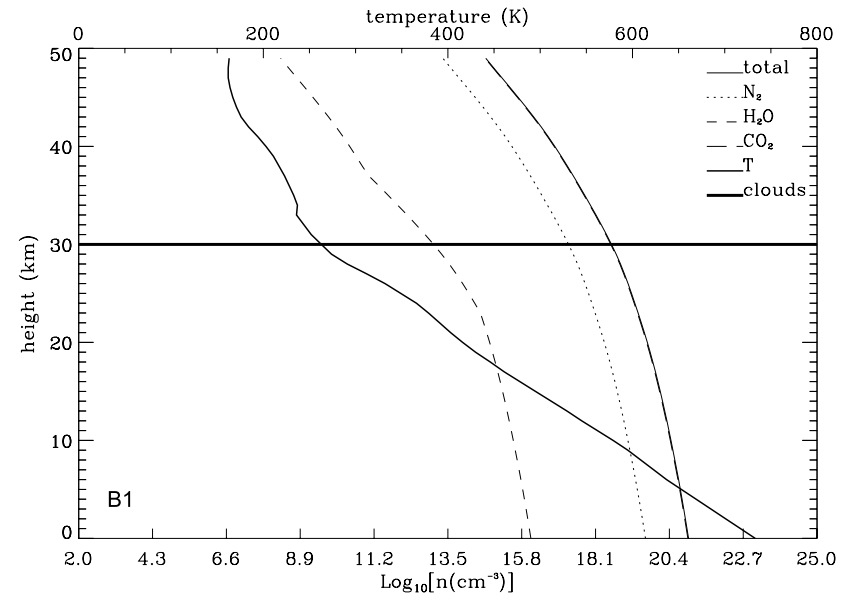

Fig. 3. Atmospheric profiles, B1. The legend is identical to Fig. 2. The temperature profile and mixing ratios are those of Venus. The temperature is considered to be constant above $50 \mathrm{~km}$. Carbon dioxide is barely visible because it is by far the major constituent, so its line is superimposed on the total density line.

\subsubsection{Presence of clouds}

In the wavelength range of interest, the surface of Venus is almost completely hidden by clouds; therefore, it seems reasonable to model these types of clouds to a first order

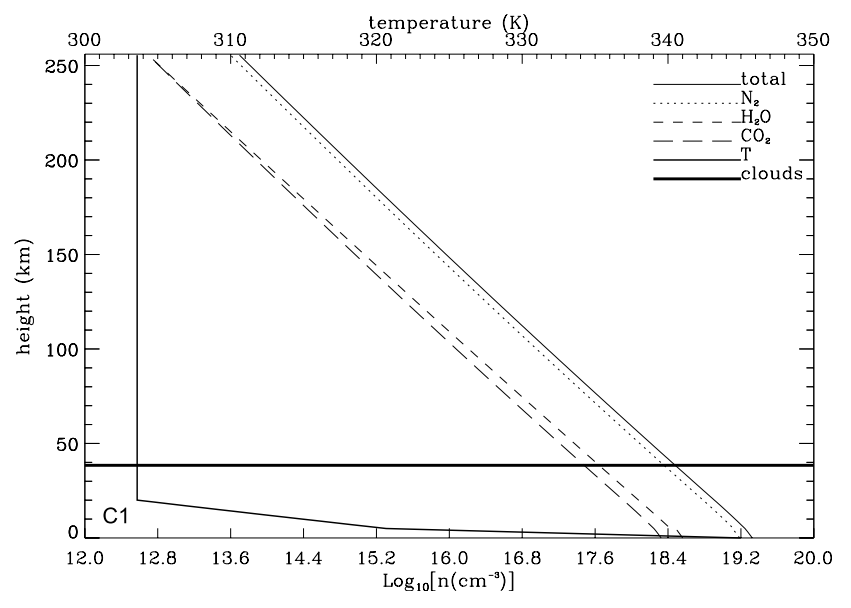

Fig. 4. Atmospheric profiles, C1. Same legend as in Figs. 2 and 3. The temperature profile follows a dry adiabat in the first $10 \mathrm{~km}$ of the atmosphere, until the point where $e \geq e_{\text {sat }}$. Next, it follows a steeper saturated adiabat up to $20 \mathrm{~km}$ high. The temperature gradient is arbitrarily set to be isothermal above this point. The cloud top (thickest line) is one scale-height above the higher point where $e \geq e_{\text {sat }}$. For reasons detailed in the text (see Sect. 2.2.5), this point corresponds to the level where the temperature gradient becomes isothermal.

approximation by assuming that they act as an optically thick layer at a given altitude. As a result, clouds effectively increase the apparent radius of the planet, and the transiting spectrum gives information only about the atmospheric components existing above the cloud layer. The top of the cloud layer is a free parameter for $\mathrm{N}_{2} / \mathrm{O}_{2}$ - and $\mathrm{CO}_{2}$-rich atmospheres, set to 10 and $30 \mathrm{~km}$, taken from the Earth and Venus, respectively. We treat the case of the $\mathrm{N}_{2} / \mathrm{H}_{2} \mathrm{O}$-rich atmosphere separately because $\mathrm{H}_{2} \mathrm{O}$ is a highly condensable species.

\subsubsection{Composition, vertical structure and location of the clouds in a $\mathrm{N}_{2} / \mathrm{H}_{2} \mathrm{O}$-rich atmosphere}

The temperature gradient of an atmosphere containing a nonnegligible amount of condensable species, like $\mathrm{H}_{2} \mathrm{O}$, significantly departs from the case where no condensation occurs. A correct estimation of the temperature profile is crucial to determining the scale height, hence the detectability of that atmosphere. In an $\mathrm{H}_{2} \mathrm{O}$-rich atmosphere, the evolution of the adiabatic temperature gradient is driven by the ratio of the partial pressure of water vapor, $e$, to the saturating vapor pressure, $e_{\mathrm{sat}}$. This ratio should also determine the levels at which the water vapor is in excess in the air and condenses (for $e / e_{\text {sat }}>1$ ), i.e. the levels where clouds may form. 
Table 2. Summary of test models.

\begin{tabular}{cccccccccc}
\hline \hline Model & Description & Atm. type & $\begin{array}{c}R_{\mathrm{P}} \\
\left(R_{\oplus}\right)\end{array}$ & $\begin{array}{c}M_{\mathrm{P}} \\
\left(M_{\oplus}\right)\end{array}$ & $\begin{array}{c}\rho_{\mathrm{P}} \\
\left(\mathrm{g} \mathrm{cm}^{-3}\right)\end{array}$ & $\begin{array}{c}g \\
\left(\mathrm{~m} \mathrm{~s}^{-2}\right)\end{array}$ & $\begin{array}{c}p_{0} \\
(\mathrm{~atm})\end{array}$ & $\begin{array}{c}H_{0} \\
(\mathrm{~km})\end{array}$ & $\begin{array}{c}h_{\max } \\
(\mathrm{km})\end{array}$ \\
\hline $\mathrm{A} 1$ & $(\approx)$ Earth & $\mathrm{N}_{2} / \mathrm{O}_{2}$-rich & 1 & 1 & 5.5 & 9.8 & 1 & 8.8 & 85 \\
$\mathrm{~B} 1$ & $(\approx)$ Venus & $\mathrm{CO}_{2}$-rich & 1 & 1 & 5.5 & 9.8 & 100 & 14.3 & 50 \\
$\mathrm{C} 1$ & medium ocean-planet & $\mathrm{N}_{2} / \mathrm{H}_{2} \mathrm{O}$-rich & 1 & 0.5 & 2.8 & 4.9 & 1 & 20.0 & 260 \\
$\mathrm{~A} 2$ & small Earth & $\mathrm{N}_{2} / \mathrm{O}_{2}$-rich & 0.5 & 0.1 & 4.0 & 3.9 & 1 & 24.7 & 260 \\
$\mathrm{~B} 2$ & small Venus & $\mathrm{CO}_{2}$-rich & 0.5 & 0.1 & 4.0 & 3.9 & 1 & 40.0 & 99 \\
$\mathrm{C} 2$ & small ocean-planet & $\mathrm{N}_{2} / \mathrm{H}_{2} \mathrm{O}$-rich & 0.5 & 0.05 & 1.8 & 2.0 & 1 & 61.4 & 499 \\
$\mathrm{~A} 3$ & "super-Earth" & $\mathrm{N}_{2} / \mathrm{O}_{2}$-rich & 2 & 9 & 6.1 & 24.5 & 1 & 3.9 & 30 \\
$\mathrm{~B} 3$ & "super-Venus" & $\mathrm{CO}_{2}$-rich & 2 & 6 & 6.1 & 24.5 & 100 & 6.4 & 30 \\
$\mathrm{C} 3$ & big ocean-planet & $\mathrm{N}_{2} / \mathrm{H}_{2}$ O-rich & 2 & 9 & 4.1 & 14.7 & 1 & 6.7 & 60 \\
\hline
\end{tabular}

Our initial conditions at the $z=0$ level $\left(z^{0}\right)$ are temperature $T^{0}$ and pressure $p^{0}$. With these quantities we can estimate $e_{\text {sat }}$, which depends only on the temperature, using the Clausius-Clapeyron equation:

$e_{\text {sat }}(T)=p^{*} \exp \left[\frac{\mu_{\mathrm{H}_{2} \mathrm{O}} L_{v}}{\mathcal{N}_{\mathrm{A}} k}\left(\frac{1}{T^{*}}-\frac{1}{T}\right)\right]$

where $p^{*}$ and $T^{*}$ are the reference pressure $\left(1.013 \times 10^{5} \mathrm{~Pa}\right)$ and temperature $(373 \mathrm{~K}), \mu_{\mathrm{H}_{2} \mathrm{O}}$ is the molar mass of water, and $L_{v}$ is the latent heat of vaporization for water $\left(2.26 \times 10^{10} \mathrm{erg} \mathrm{g}^{-1}\right)$. Assuming that the planet is covered with liquid water (e.g., an ocean-planet; see Léger et al. 2004) and that $T^{0}$ is "tropical" (e.g. $340 \mathrm{~K}$ ), the humidity at the surface is high so that the value of $e^{0}$ must be an important fraction of $e_{\text {sat }}\left(T^{0}\right)$. We set $e^{0}$ to half the value of $e_{\mathrm{sat}}\left(T^{0}\right)$. The volume-mixing ratio of water can be expressed as $Y_{\mathrm{H}_{2} \mathrm{O}}=e / p$, and we can calculate it at the surface of the planet. The atmosphere of an "ocean-planet" may also contain a significant quantity of $\mathrm{CO}_{2}$. We arbitrarily set this quantity constant to $Y_{\mathrm{CO}_{2}}=0.1$ (Léger et al. 2004; Ehrenreich et al. 2005b). Molecular nitrogen is the major constituent of the atmosphere of the Earth and the second more abundant species in the atmosphere of Venus; therefore, we chose to include it to complete the chemical composition of this atmosphere. The mixing ratio of $\mathrm{N}_{2}$ was set to be $Y_{\mathrm{N}_{2}}=1-Y_{\mathrm{CO}_{2}}-Y_{\mathrm{H}_{2} \mathrm{O}}$ at any level. Assuming the atmosphere contains only $\mathrm{N}_{2}, \mathrm{H}_{2} \mathrm{O}$ and $\mathrm{CO}_{2}$, we can obtain the mean molar mass of the atmospheric gas $\left(\mu^{0}=\sum_{i} Y_{i}^{0} \mu_{i}\right)$ and that of the dry atmospheric gas $\left(\mu_{\mathrm{d}}^{0}=\mu^{0}-Y_{\mathrm{H}_{2} \mathrm{O}}^{0} \mu_{\mathrm{H}_{2} \mathrm{O}}\right)$, the mean specific heat of dry air $\left(C_{p}^{0}=\sum C_{p_{i}} Y_{i}^{0} \mu_{i} / \mu_{\mathrm{d}}^{0}\right)$ and the scale height $H_{0}$ (all at the level $\left.z^{0}\right)$.

For the $z^{j+1}$ level, we needed to evaluate the temperature gradient between $z^{j}$ and $z^{j+1}$. There are two cases (Triplet $\&$ Roche 1986):

$-e^{j}<e_{\text {sat }}^{j} ;$ in this case the temperature follows a dry adiabatic gradient,

$$
\Delta T_{\mathrm{dry}}=\frac{-g}{C_{p}^{j}} .
$$

- $e^{j}=e_{\text {sat }}^{j} ;$ in this case the gradient is saturated,

$$
\Delta T_{\text {sat }}=\Delta T_{\text {dry }} \frac{\left(1+r_{\text {sat }}^{j}\right)\left[1+L_{v} r_{\text {sat }}^{j} /\left(R_{\mathrm{dry}}^{j} T^{j}\right)\right]}{1+\frac{r_{\text {sat }}^{j}}{C_{p}^{j}}\left[C_{p_{\mathrm{H}_{2} \mathrm{O}}}+L_{v}^{2} \frac{1+r_{\text {sat }}^{j} R_{\mathrm{H}_{2} \mathrm{O}} R_{\mathrm{dry}}^{j}}{R_{\mathrm{H}_{2} \mathrm{O}}\left(T^{j}\right)^{2}}\right]}
$$

where $r_{\text {sat }}^{j}=\left(\mu_{\mathrm{H}_{2} \mathrm{O}} e_{\mathrm{sat}}^{j}\right) /\left[\mu_{\mathrm{d}}^{j}\left(p^{j}-e_{\text {sat }}^{j}\right)\right]$ is the mixing ratio of saturated air, $R_{\text {dry }}^{j}=\mathcal{N}_{\mathrm{A}} k / \mu_{\text {dry }}^{j}$, and $R_{\mathrm{H}_{2} \mathrm{O}}=\mathcal{N}_{\mathrm{A}} k / \mu_{\mathrm{H}_{2} \mathrm{O}}$ are the specific constant of dry air at the level $z^{j}$ and water (respectively).

If $z^{j+1}<20 \mathrm{~km}$, we select the appropriate gradient accordingly to the value of $e / e_{\text {sat }}$, and get the value of the temperature $T^{j+1}$. Above $20 \mathrm{~km}$, we assumed the temperature profile becomes isothermal $\left(T^{j+1}=T^{j}\right)$.

The assumption of an isothermal atmosphere, already discussed in Sect. 2.2.2, is somewhat arbitrary but is motivated by an analogy with the atmosphere of the Earth, where the temperature gradient becomes positive above about $20 \mathrm{~km}$ and up to $50 \mathrm{~km}$. Taking an isothermal temperature gradient will conservatively mimic the presence of a stratosphere. However, it has important consequences, since it allows $\mathrm{H}_{2} \mathrm{O}$ to be present in significant amounts above the cloud top. In fact, above $20 \mathrm{~km}$, the temperature stops decreasing, preventing condensation from occurring as the saturation vapor pressure depends only on temperature. Our assumption consequently fixes the height of the cloud deck to the point where the temperature profile is isothermal (actually, one scale height above that point). If we set this point higher, we would increase the amount of clouds, thereby reducing the detectable portion of the atmosphere.

We calculated $H^{j+1}, p^{j+1}=p^{j} \cdot \exp \left(-z^{j+1} / H^{j+1}\right), e_{\text {sat }}^{j}$ (from Eq. (5)), and either $\left.e^{j+1}=e^{j} \cdot \exp \left[\left(z^{j}-z^{j+1}\right) / H^{j+1}\right)\right]$, if the atmosphere is not saturated, or $e^{j+1}=e_{\text {sat }}^{j+1}$, if the atmosphere is saturated. We finally found all $Y_{i}^{j+1}, \mu_{\text {dry }}^{j+1}$ and $C_{p_{\text {dry }}}^{j+1}$ and then iterated the process for all atmospheric levels.

The higher and the lower pressure levels, where $e=e_{\text {sat }}$ indicate, respectively, the bottom and the top of the region where clouds are forming. We assumed the cloud layer does not extend over one scale height above the top of the cloud-forming region. However, we can still have $e \leq e_{\text {sat }}$ higher in the atmosphere, and thus $\mathrm{H}_{2} \mathrm{O}$ can be present above the clouds.

\subsection{Description of atmospheric absorptions}

\subsubsection{Chemical species}

We used the program LBLABC (Meadows \& Crisp 1996), a line-by-line model that generates monochromatic gas absorption coefficients from molecular line lists, for each of the gases 


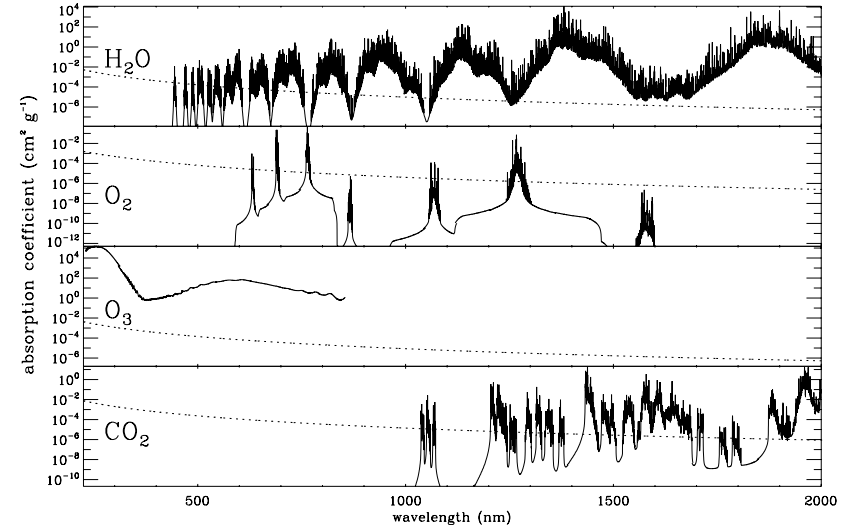

Fig. 5. Absorption coefficients of atmospheric absorbers (in $\mathrm{cm}^{2} \mathrm{~g}^{-1}$ ), as a function of the wavelength. The photo-absorption coefficients corresponding to $\mathrm{H}_{2} \mathrm{O}, \mathrm{O}_{2}, \mathrm{O}_{3}$, and $\mathrm{CO}_{2}$ (solid lines) are plotted against their respective Rayleigh-scattering coefficient (dotted line), except $\mathrm{O}_{3}$, plotted against the Rayleigh-scattering coefficient of $\mathrm{N}_{2}$.

except ozone that are present in the atmosphere. The line lists are extracted from the HITRAN 2000 databank (Rothman et al. 2003). We calculated the absorption coefficients for $\mathrm{O}_{2}$, $\mathrm{H}_{2} \mathrm{O}$, and $\mathrm{CO}_{2}$ in our wavelength range we (i.e., from 200 to $2000 \mathrm{~nm}$ ).

The absorption coefficients relative to these species depend on pressure and temperature. We verified that those variations do not have a significant impact on the results (see Sect. 4) and decided to use the absorption coefficients calculated at the pressure and temperature of the cloud layer, i.e., $10 \mathrm{~km}$ in models $\mathrm{A} 1$ to $\mathrm{A} 3,30 \mathrm{~km}$ in models $\mathrm{B} 1$ to $\mathrm{B} 3$, and from 25 to $70 \mathrm{~km}$ in models $\mathrm{C} 1$ to $\mathrm{C} 3$. We then assumed these absorption coefficients to be constant along the $z$-axis. This is a fairly good approximation, since molecules at that atmospheric level contribute more substantially to the transmitted spectrum than molecules at the bottom of the atmosphere. Absorption coefficients for $\mathrm{H}_{2} \mathrm{O}, \mathrm{CO}_{2}, \mathrm{O}_{3}$, and $\mathrm{O}_{2}$ are compared in Fig. 5.

The spectrum of $\mathrm{O}_{3}$ is unavailable in HITRAN at wavelengths lower than $2.4 \mu \mathrm{m}$. However it has strong absorption in the Hartley (200-350 nm) and Chappuis (400-750 nm) bands. Thus we took the photo-absorption cross-sections, $\sigma$ (in $\mathrm{cm}^{2}$ ), from the GEISA/cross-sectional databank (Jacquinet-Husson et al. 1999) and converted them into absorption coefficients, $A$ (in $\mathrm{cm}^{2} \mathrm{~g}^{-1}$ ), such as $A=\sigma \mathcal{N}_{\mathrm{A}} / \mu$, where $\mu$ is the molar mass of the component.

As shown in Fig. 6, the pressure and the temperature variations do not significantly influence the cross sections/absorption coefficients of $\mathrm{O}_{3}$. We therefore used the values given for $p=1 \mathrm{~atm}^{3}$ and $T=300 \mathrm{~K}$, and set them constant along the $z$-axis.

\subsubsection{Rayleigh diffusion}

Light is scattered toward short wavelengths by atmospheric molecules whose dimensions are comparable to $\lambda$. Rayleigh diffusion could be an important indicator of the most abundant atmospheric species. Molecular nitrogen, for instance,

\footnotetext{
$31 \mathrm{~atm}=1013 \mathrm{hPa}$.
}

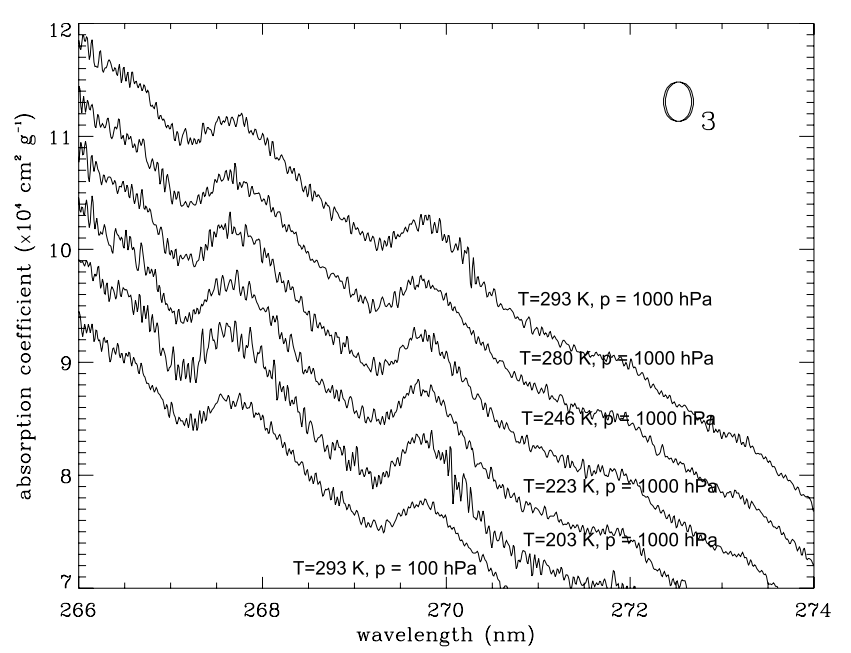

Fig. 6. Dependence of the absorption coefficient of $\mathrm{O}_{3}$ on pressure and temperature. For clarity, each line has been shifted down by $5 \times 10^{4} \mathrm{~cm}^{2} \mathrm{~g}^{-1}$ with respect to the previous one.

does not present any noticeable spectroscopic lines between 0.2 and $2 \mu \mathrm{m}$. With a transit observation, the presence of a gas without spectroscopic lines like nitrogen in the Earth atmosphere can be indirectly inferred from the wavelength-dependence of the spectrum ratio continuum. Since the Rayleigh-scattering cross section of $\mathrm{CO}_{2}$ is high, Venus-like atmospheric signatures should also present a significantly large Rayleigh-scattering contribution.

We have therefore estimated all of these different contributions. The Rayleigh-scattering cross section, $\sigma_{\mathrm{R}}$, can be expressed in cgs units as (Bates 1984; Naus \& Ubachs 1999; Sneep \& Ubachs 2004)

$\sigma_{\mathrm{R}}(\bar{v})=\frac{24 \pi^{3} \bar{v}^{4}}{n^{2}}\left(\frac{r(\bar{v})^{2}-1}{r(\bar{v})^{2}+2}\right)$,

where $\bar{v}=1 / \lambda, n$ is the number density $\left(\mathrm{cm}^{-3}\right)$ and $r$ is the refractive index of the gas. The total Rayleigh scattering includes weighted contributions from $\mathrm{N}_{2}, \mathrm{O}_{2}, \mathrm{CO}_{2}$, and $\mathrm{H}_{2} \mathrm{O}$ (i.e., $\sigma_{\mathrm{R}}=\sum_{i} Y_{i} \sigma_{\mathrm{R} i}$ ), so we need all the corresponding refractive indices. These are found in Bates (1984) and Sneep \& Ubachs (2004) for $\mathrm{N}_{2}, \mathrm{O}_{2}$ and $\mathrm{CO}_{2}{ }^{4}$. The refractive index for $\mathrm{H}_{2} \mathrm{O}$ comes from Schiebener et al. (1990). Tests have proved that the different refractive indexes do not significantly change with temperature and pressure. We therefore calculated the indexes for standard conditions $\left(15^{\circ} \mathrm{C}\right.$ and $\left.1013 \mathrm{hPa}\right)$.

\subsubsection{Refraction}

Depending on the wavelength, the refraction may bring rays coming from different parts of the star into the line of sight. To quantify the importance of that effect, we calculated the maximum deviation, $\Delta \theta$, due to the wavelength dependence of the refraction index, using the formula given by

${ }^{4}$ We call the reader's attention to a typographical error in the $\mathrm{CO}_{2}$ refractive index formula (Eq. (13)) in Sneep \& Ubachs (2004): in order to yield the correct values, results from this expression should be divided by $10^{3}$ (M. Sneep, personal communication). 
Seager \& Sasselov (2000) and the refractive index at the surface $(h=0)$ between 0.2 and $2 \mu \mathrm{m}$. We obtained $\Delta \theta \approx 0.3^{\prime}$, which represents about $1.5 \%, 1 \%$, and $0.5 \%$ of the angular diameter of the star (F-, G-, and K-type star, respectively) as seen from the planet. We therefore consider this effect negligible, as long as there are no large variations in the stellar flux on scales lower than the surface corresponding to these numbers.

\subsection{Choice of test models}

We chose 9 cases, divided into 3 categories: $1 R_{\oplus}$-planets (models $\mathrm{A} 1, \mathrm{~B} 1$ and $\mathrm{C} 1), 0.5 R_{\oplus}$-planets (A2, B2, and C2) and $2 R_{\oplus}$-planets (A3, B3, and C3). The parameters for each model are summarized in Table 2. For theses ranges of planetary radii, the depth of the occultation by the tested planets will differ by a factor of $\sim 16$ at most during their transit. Notice that better detection of the transit itself does not always imply better detection for the atmosphere of the transiting planet. On the contrary, in some cases, the fainter the transit is, the more detectable the atmosphere will be! In any case, we naturally need to secure the detection of the planet itself before looking for an atmosphere.

The choice of studying planets with a variety of sizes gives us the possibility to explore a large range of planet characteristics, including mass, radius, and density. The Earth density is $5.5 \mathrm{~g} \mathrm{~cm}^{-3}$. A planet with the internal composition of the Earth and twice its radius would weigh $\sim 10$ times more, while a planet half as large would weigh $\sim 10$ times less (Sotin et al. 2005). That gives densities of 6.1 and $4 \mathrm{~g} \mathrm{~cm}^{-3}$, respectively. We thus have 3 cases, each of which can be coupled with a plausible atmosphere. We chose an $\mathrm{N}_{2} / \mathrm{O}_{2}$-rich atmosphere (similar to that of the Earth) for models A1, A2, and A3, and a Cytherean (i.e., Venus-like ${ }^{5}$ ) $\mathrm{CO}_{2}$-rich atmosphere for models B1, B2, and B3.

Note that the atmospheric pressure profiles were scaled from the $1 R_{\oplus}$ cases (A1 and $\mathrm{B} 1$ ) to the 0.5 and $2 R_{\oplus} \bmod$ els. In doing so, we did not include any species that showed a peak of concentration in altitude, such as the $\mathrm{O}_{3}$ layer in model A1. In fact, the $\mathrm{O}_{3}$ peak not only depends on the hydrostatic equilibrium, but also on the photochemical equilibrium at the tropopause of the Earth. For that reason $\mathrm{O}_{3}$ is absent in models A2 and A3.

Léger et al. (2004) suggest the existence of "ocean-planets" whose internal content in volatiles $\left(\mathrm{H}_{2} \mathrm{O}\right)$ might be as high as $50 \%$ in mass. Such planets would be much less dense than telluric ones. We are particularly interested in those ocean-planets since the lower the density of the planet is, the higher the atmosphere extending above the surface. These objects could have densities of $1.8,2.8$, and $4.1 \mathrm{~g} \mathrm{~cm}^{-3}$ for radii of $0.5,1$, and $2 R_{\oplus}$ (Sotin et al. 2005), which are relatively small, but reasonable if compared with Titan's density $\left(1.88 \mathrm{~g} \mathrm{~cm}^{-3}\right)$. The huge quantity of water on the surface of an ocean-planet could produce a substantial amount of water vapor in their atmosphere, if the temperature is high enough. A non-negligible

\footnotetext{
${ }^{5}$ Cythera $(K \dot{v} \theta \eta \rho \alpha)$ is an Ionian island where, according to the Greek mythology, the goddess Aphrodite/Venus first set foot. See http://en.wikipedia.org/wiki/Cytherean
}

concentration of $\mathrm{CO}_{2}$ might be present, as well, in those atmospheres (Ehrenreich et al. 2005b). Using this information on ocean-planets, we can simulate three extra cases, namely $\mathrm{C} 1$, C2, and C3 (Table 2).

\subsection{Choice of different stellar types}

In this work, we consider planets orbiting in the habitable zone (HZ) of their parent star. Our atmospheric models are not in fact a good description for planets orbiting too close to their parent star. For instance, the heating of the atmosphere by an extremely close star could trigger effects like evaporation, thus invalidating the hydrostatic equilibrium we have assumed (see, for instance, Lecavelier des Etangs et al. 2004; Tian et al. 2005). The reduced semi-major axis $a_{\mathrm{r}}$ of the orbit of all planets we have considered is defined as:

$a_{\mathrm{r}}=a \cdot\left(L_{\star} / L_{\odot}\right)^{-0.5}$.

We set $a_{\mathrm{r}}=1$ astronomical unit (AU), so that the planet is in the $\mathrm{HZ}$ of its star.

Here we focus on Earth-size planets orbiting different main sequence stars, such as K-, G-, and F-type stars, since the distribution of stellar photons in the spectrum is different from one spectral type to another. Planets in the $\mathrm{HZ}$ of $\mathrm{K}, \mathrm{G}$, and $\mathrm{F}$ stars, with $a_{\mathrm{r}}=1 \mathrm{AU}$, should have a real semi-major axis of $0.5,1$, and $2 \mathrm{AU}$, respectively.

\section{Signal-to-noise ratio for ideal observations}

Prior to the atmospheres, we need to detect the planets themselves with a dedicated survey, as the one proposed by Catala et al. (2005). The transmission spectroscopy we theoretically study here requires the use of a large space telescope. Hence, we need to quantify the $S / N$ of such observations to determine the detectability of the atmospheric signatures for a transiting Earth-size exoplanet. The $S / N$ will depend on both instrumental and astrophysical parameters.

\subsection{Instrumental requirements}

The first relevant parameter related to the instrumentation is the effective area of the telescope collecting mirror, $S$, which can be expressed as $S=(\epsilon D)^{2} \pi / 4$. The coefficient $\epsilon^{2}$ accounts for the instrumental efficiency, so $\epsilon D$ is the "effective diameter" of the mirror. Up to now, all exoplanetary atmospheric signatures have been detected by the Space Telescope Imaging Spectrograph (STIS) onboard the Hubble Space Telescope (HST). This instrument, now no longer operative, was very versatile $^{6}$ and consequently was not built to have high efficiency. It had a throughput $\epsilon^{2} \approx 2 \%$ from 200 to $300 \mathrm{~nm}$, and $\epsilon^{2} \approx 10 \%$ from 350 to $1000 \mathrm{~nm}$. As the majority of photons we are interested in are available in the range from 350 to $1000 \mathrm{~nm}$, we reasonably assume that a modern spectrograph has a mean $\epsilon^{2}$ significantly greater than $10 \%$ from 200 to $2000 \mathrm{~nm}$. Presently the most efficient spectrographs have $\epsilon^{2} \approx 25 \%$ in the visible,

\footnotetext{
6 STIS was used for imagery, spectro-imagery, coronography, and low and high resolution spectroscopy.
} 


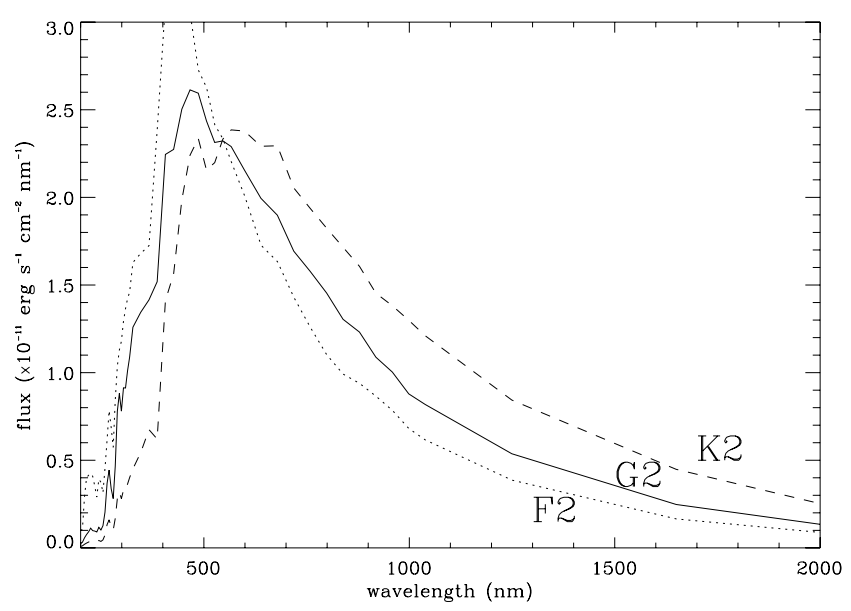

Fig. 7. Spectrum of a K2 (dashed line), G2 (solid line), and F2-type stars (dotted line) between 0.2 and $2 \mu \mathrm{m}$. The fluxes are scaled to an apparent magnitude $V=8$.

so it seems reasonable to imagine that next-generation spectrographs, specifically designed to achieve high sensitivity observations, could have a throughput of $\epsilon^{2} \approx 25 \%$, or $\epsilon=50 \%$.

Another parameter linked to the instrument is the spectral resolution, $\mathcal{R}$. In the following, $\mathcal{R}$ will be assumed to be about 200, i.e. a $10 \mathrm{~nm}$-wide spectral feature can be resolved.

Finally, it is legitimate to question the ability of the instrument detectors to discriminate the tenuous $\left(\sim 10^{-6}\right)$ absorption features in the transmitted spectra of Earth-size planets. In the recent past, sodium was detected at a precision of 50 parts-permillion (ppm) on a line as thin as about $1 \mathrm{~nm}$ by Charbonneau et al. (2002) using STIS. According to our results (see Sect. 4), some absorption features from Earth-size planet atmospheres show a $\sim 1$ ppm dimming over $\sim 100 \mathrm{~nm}$ : the technological improvement required to fill the gap should not be unachievable. Besides, since we are dealing with relative measurements the in-transit signal being compared to the out-of-transit one - there is no need to have detectors with a perfect, absolute calibration. A highly stable response over periods of several hours is all that is truly required. Nevertheless, instrumental precision remains a challenging issue whose proper assessment will require further, detailed studies.

\subsection{Physical constraints on the observation}

The number of photons detected as a function of wavelength depends on the spectral type of the star, while the total number of photons received in an exposure of duration $t$ depends on the apparent magnitude of the star, $V$. The stellar spectra $F_{\star}^{V=0}(\lambda)$ are from $\rho$ Capricorni (F2 IV), HD 154760 (G2 V), and HD 199580 (K2 IV) and are taken from the BruzualPersson-Gunn-Stryker (BPGS) spectrophotometry atlas ${ }^{7}$. The fluxes ( $\mathrm{erg} \mathrm{cm}^{-2} \mathrm{~s}^{-1} \AA^{-1}$ ) are given at a null apparent magnitude, so we re-scaled them for any apparent magnitude $V$, $F_{\star}=F_{\star}^{V=0} \cdot 10^{-0.4 V}$. The three corresponding spectra are plotted for a default magnitude $V=8$ in Fig. 7 .

\footnotetext{
${ }^{7}$ Available on ftp.stsci.edu/cdbs/cdbs2/grid/bpgs/
}

The stellar type determines the radius and the mass of the star, so the transit duration (and thus the maximum time of exposure during the transit) is different depending on the star we consider. The transit duration is also a function of the semimajor axis of the planet orbit. Since we chose a constant reduced distance $\left(a_{\mathrm{r}}=1 \mathrm{AU}\right)$ for all planetary models (see Sect. 2.5), the duration of transit depends on the stellar luminosity as well. From Zombeck (1990), we obtain the radii of $\mathrm{F}$ and $\mathrm{K}$ stars relative to that of the Sun $\left(R_{\mathrm{F}} / R_{\odot} \approx 1.25\right.$ and $R_{\mathrm{K}} / R_{\odot} \approx 0.75$, respectively $)$, the mass ratios $\left(M_{\mathrm{F}} / M_{\odot} \approx 1.75\right.$ and $\left.M_{\mathrm{K}} / M_{\odot} \approx 0.5\right)$, and the luminosity ratios $\left(L_{\mathrm{F}} / L_{\odot} \approx 4\right.$ and $\left.L_{\mathrm{K}} / L_{\odot} \approx 0.25\right)$. Using Eq. (9), the duration of the transit is:

$\tau \approx \frac{13 \pi}{4} \mathrm{~h} \cdot \frac{R_{\star}}{R_{\odot}}\left(\frac{M_{\star}}{M_{\odot}}\right)^{-0.5}\left(\frac{L_{\star}}{L_{\odot}}\right)^{0.25}$,

where $13 \pi / 4 \mathrm{~h}$ is the mean transit duration of a planet at $1 \mathrm{AU}$, averaged over all possible impact parameters of the transit across a G star. From Eq. (10) we obtain mean transit durations of 7.6, 10.2, and $13.6 \mathrm{~h}$ for K-, G-, and F-type star, respectively. In the following, we set $t=\tau$.

Ideally, our observations are limited only by the stellar photon noise; the detection of sodium at a precision of $\sim 50 \mathrm{ppm}$ in the atmosphere of HD 209458 by bharbonneau et al. (2002) was in fact limited by the stellar photon noise. However, at the low signal levels we are seeking, the intrinsic stellar noise might need to be considered as well. Stellar activity, as well as convective motions, will cause variations in both intensity and color in the target stars, on a large variety of timescales.

The impact of stellar micro-variability on the detectability of photometric transits has been addressed by a number of studies (see, e.g., Moutou et al. 2005; Aigrain et al. 2004, especially their Fig. 8; Lanza et al. 2004), all pointing towards photometric variability levels in the range of $\sim 100-1000 \mathrm{ppm}$ for durations of a few days. This is to be compared to the strength and duration of the atmospheric signatures we want to look at: $\sim 1$ ppm variations lasting a few hours. While the different time frequency and spectral content of these signatures versus the stellar noise will hopefully allow the two to be distinguished, the impact of stellar micro-variability on such faint signals is likely to be significant and may limit the ability to detect an atmosphere in a transiting planet. For instance, Aigrain et al. (2004) suggest that K stars are more adapted than $\mathrm{G}$ or F stars regarding the detection of terrestrial planets versus stellar micro-variability.

However, note that the observation of several transits for each planet considered will confirm the signal detected in the first transit. For instance, at $a_{\mathrm{r}}=1 \mathrm{AU}$ around a $\mathrm{K}$ star, a planet has a period of $\approx 0.3 \mathrm{yr}$, allowing several transit observations to be scheduled within a short period of time. Finally, the usual technique for detecting a spectral signature from a transit is to compare in-transit and out-of-transit observations (Vidal-Madjar et al. 2003, 2004). For all these reasons, we will assume in the following the ability to discriminate a transit signal from the stellar activity, and consequently the photon-noise will be the limiting factor. Nevertheless, further, detailed analysis is certainly needed to quantify the effect of stellar microvariability as a function of the stellar type, but this is outside the scope of this paper. 


\subsection{Calculation of the signal-to-noise ratio}

Now let $\varphi_{\star}$ be the maximum number of photons per element of resolution that can be received during $\tau$ : $\varphi_{\star}=F_{\star}(\lambda) \cdot \lambda /\left(h_{\mathrm{P}} c\right) \cdot \mathcal{R} \cdot S \cdot \tau$, where $h_{\mathrm{P}}$ is Planck's constant and $c$ the speed of light. Some photons are blocked or absorbed by the planet, therefore the actual number of photons received during the transit is $\varphi=\varphi_{\star}\left(1+\mathfrak{R}^{\prime}\right)$ per element of resolution.

From the observations, it is possible to obtain $\tilde{R}_{\mathrm{P}}$, an estimate of the radius of the transiting planet $R_{\mathrm{P}}$ (e.g., by using the integrated light curve or a fit to the observed spectrum ratio). This value corresponds to the flat spectrum ratio (i.e., a planet without atmosphere) that best fits the data. The corresponding number of photons received during an observation per element of resolution is therefore expressed as: $\tilde{\varphi}=\varphi_{\star}\left[1-\left(\tilde{R}_{\mathrm{P}} / R_{\star}\right)^{2}\right]$.

The weighted difference between $\varphi$ and $\tilde{\varphi}$ can reveal the presence or absence of a planetary atmosphere. We express the $\chi^{2}$ of this difference over all the elements of resolution $k$ as $\sum_{k}\left[\left(\varphi_{k}-\tilde{\varphi}_{k}\right) / \sigma_{\varphi_{k}}\right]^{2}$. Here, the uncertainty of the number of photons received is considered to be dominated by the stellar photon noise (see Sect. 3.2), that is, $\sigma_{\varphi}=\sqrt{\varphi}$. We thus have:

$\chi^{2}=\sum_{k}\left(\frac{\varphi_{\star}}{1+\mathfrak{R}_{k}^{\prime}}\left[\mathfrak{R}_{k}^{\prime}+\left(\tilde{R}_{\mathrm{P}} / R_{\star}\right)^{2}\right]^{2}\right)$.

Given the $\chi^{2}$, the $S / N$ can be directly calculated by taking its square root. The best estimation can be obtained by minimizing the $\chi^{2}$ with respect to the radius $\tilde{R}_{\mathrm{P}}$, i.e., $\partial \chi^{2} / \partial \tilde{R}_{\mathrm{P}}=0$. From this formula we can calculate the estimated radius:

$\tilde{R}_{\mathrm{P}}=R_{\star} \sqrt{-\frac{\sum_{k}\left[\varphi_{\star} \mathfrak{R}_{k}^{\prime} /\left(1+\mathfrak{R}_{k}^{\prime}\right)\right]}{\sum_{k}\left[\varphi_{\star k} /\left(1+\mathfrak{R}_{k}^{\prime}\right)\right]}}$.

Once we determine if an atmosphere is observable or not (depending on the $S / N$ ratio), we can use a similar approach to quantify the detectability of the single atmospheric absorber contributing to the total signal $\varphi$. Let $\hat{\varphi}_{i}=\varphi_{\star}\left(1+\hat{\mathfrak{R}}_{i}^{\prime}\right)$ be the signal obtained by filtering the stellar light out of all atmospheric absorbers except the $i$ th, and let $\left(\tilde{\hat{\varphi}}_{i}\right)$ be its estimation. Here, $\hat{\mathfrak{R}}_{i}^{\prime}$ is the spectrum ratio calculated when the species $i$ is not present in the atmosphere. Further, since $\left(\tilde{\hat{\varphi}}_{i}\right) \approx \alpha_{i} \hat{\varphi}_{i}$, we can deduce the presence of absorber $i$ in the atmosphere by simply comparing the fit we made while assuming its absence, $\alpha_{i} \hat{\varphi}_{i}$, with the measured signal $\varphi$ :

$\chi_{i}^{2}=\sum_{k}\left(\frac{\varphi_{\star k}}{1+\mathfrak{R}_{k}^{\prime}}\left[\left(1+\mathfrak{R}_{k}^{\prime}\right)-\alpha_{i}\left(1+\hat{\mathfrak{R}}_{i k}^{\prime}\right)\right]^{2}\right)$,

where

$\alpha_{i}=\frac{\sum_{k}\left[\varphi_{\star k}\left(1+\hat{\mathfrak{R}}_{i k}^{\prime}\right)\right]}{\sum_{k}\left[\varphi_{\star k}\left(1+\hat{\mathfrak{R}}_{i k}^{\prime}\right)^{2} /\left(1+\mathfrak{R}_{k}^{\prime}\right)\right]}$.

\section{Results and discussion}

The results of our computations are displayed in Tables 3 and 4 and plotted as spectrum ratios in Figs. 8-10.

\subsection{Spectral features of interest}

Here we summarize the contributions of each atmospheric absorber to the spectrum ratio for various models. The spectral resolution of the plots presented here is $10 \mathrm{~nm}$. The most prominent spectral signatures, when present, are those of $\mathrm{O}_{3}$ and $\mathrm{H}_{2} \mathrm{O}$. Carbon dioxide is hard to distinguish from $\mathrm{H}_{2} \mathrm{O}$ bands and/or its own Rayleigh scattering. Molecular oxygen transitions are too narrow to contribute significantly to the spectrum ratio.

\subsubsection{Ozone}

In the spectral domain studied here, the Hartley (200-350 nm) and Chappuis (420-830 nm) bands of $\mathrm{O}_{3}$ appear to be the best indicators of an Earth-like atmosphere. These bands are large (150 and $600 \mathrm{~nm}$, respectively) and lie at the blue edge of the spectrum, where spectral features from other species are missing. There is no noticeable contamination by $\mathrm{H}_{2} \mathrm{O}$, while $\mathrm{O}_{2}$ strong transitions are narrow and could be separated easily. Ozone bands significantly emerge from Rayleigh scattering and correspond to very strong transitions, despite the small amount of $\mathrm{O}_{3}$ present in the model A1 atmosphere $\left(Y_{\mathrm{O}_{3}}<10^{-5}\right)$. When present, ozone is more detectable in an atmosphere that is similar to model A2.

\subsubsection{Water}

The signature of $\mathrm{H}_{2} \mathrm{O}$ is visible in a transit spectrum only if $\mathrm{H}_{2} \mathrm{O}$ is substantially abundant above the clouds. This is not the case for models with an Earth-like atmosphere like A1, A2, and A3. On the contrary, the models of the ocean-planets $(\mathrm{C} 1$, $\mathrm{C} 2$, and $\mathrm{C} 3$ ) show a major contribution from this molecule in the form of four large bands that dominate the red part of the spectrum (at $\lambda \gtrsim 950 \mathrm{~nm}$ ). For these three cases, $\mathrm{H}_{2} \mathrm{O}$ can be significantly abundant above the clouds.

\subsubsection{Carbon dioxide}

The lines of $\mathrm{CO}_{2}$ emerge about as strongly from the "continuum" as the $\mathrm{H}_{2} \mathrm{O}$ ones, but are often overlapping with these lines. The transitions around $1600 \mathrm{~nm}$ and the ones around $1950 \mathrm{~nm}$ are the easiest to identify, while other bands are not observable if water is present. Rayleigh-scattering and photoabsorption cross sections of $\mathrm{CO}_{2}$ are comparable at most wavelengths below $1.8 \mu \mathrm{m}$ (see Fig. 5), except for a few $\sim 10-\mathrm{nm}$ wide bands. In fact, the more $\mathrm{CO}_{2}$ is present in the atmosphere, the more opaque the atmosphere becomes. This implies it would be impossible for an observer on the surface of Venus to see the Sun. Carbon dioxide may be more detectable farther in the infrared, hence making further investigations up to $2.5 \mu \mathrm{m}$ desirable.

\subsubsection{Molecular oxygen}

Schneider (1994) and Webb \& Wormleaton (2001) have specifically studied the detectability of $\mathrm{O}_{2}$ during a planetary transit. Molecular oxygen does not appear in the plots of the present 
Table 3. Summary of results: signal-to-noise ratios obtainable with a telescope mirror effective size of $\epsilon D=10 \mathrm{~m}$ pointing at a $V=8$ star. To get the $S / N$ ratios for a different effective size $\epsilon D$, exposure time during transit $t$, and/or apparent magnitude of the star $V$, the result scales with $(\epsilon D / 10 \mathrm{~m}) \cdot(t / \tau)^{0.5} \cdot 10^{-0.2(V-8)}$ where $\tau$ is defined by Eq. (10). The $S / N$ by species are calculated for the models with clouds.

\begin{tabular}{|c|c|c|c|c|c|c|c|c|}
\hline \multirow[t]{2}{*}{ Model } & \multirow[t]{2}{*}{ Description } & \multirow[t]{2}{*}{ Star } & \multicolumn{6}{|c|}{$\begin{array}{l}\text { Signal-to-noise ratio } \\
(S / N)_{V=8, \epsilon D=10 \mathrm{~m}}\end{array}$} \\
\hline & & & w/o cloud & w/ clouds & $\mathrm{H}_{2} \mathrm{O}$ & $\mathrm{CO}_{2}$ & $\mathrm{O}_{3}$ & $\mathrm{O}_{2}$ \\
\hline \multirow{3}{*}{ A1 } & \multirow{3}{*}{$(\approx)$ Earth } & $\mathrm{K}$ & 5.2 & 3.5 & 1.7 & 1.1 & 1.9 & 0.2 \\
\hline & & $\mathrm{G}$ & 3.2 & 2.3 & 0.8 & 0.5 & 1.2 & 0.2 \\
\hline & & $\mathrm{F}$ & 2.3 & 1.7 & 0.5 & 0.3 & 0.9 & 0.1 \\
\hline \multirow{3}{*}{ B1 } & \multirow{3}{*}{$(\approx)$ Venus } & $\bar{K}$ & 4.0 & 2.3 & 0.0 & 2.3 & - & - \\
\hline & & $\mathrm{G}$ & 2.1 & 1.2 & 0.0 & 1.2 & - & - \\
\hline & & $\mathrm{F}$ & 1.3 & 0.7 & 0.0 & 0.7 & - & - \\
\hline \multirow{3}{*}{$\mathrm{C} 1$} & medium & $\mathrm{K}$ & 41 & 39 & 39 & 11 & - & - \\
\hline & ocean- & $\mathrm{G}$ & 22 & 20 & 20 & 5.4 & - & - \\
\hline & planet & $\mathrm{F}$ & 14 & 13 & 13 & 3.3 & - & - \\
\hline \multirow{3}{*}{$\mathrm{A} 2$} & \multirow{3}{*}{ small Earth } & $\mathrm{K}$ & 6.9 & 6.3 & 3.8 & 2.8 & - & 0.7 \\
\hline & & G & 4.3 & 4.0 & 1.8 & 1.4 & - & 0.5 \\
\hline & & $\mathrm{F}$ & 3.2 & 3.0 & 1.1 & 0.8 & - & 0.3 \\
\hline \multirow{3}{*}{ B2 } & \multirow{3}{*}{ small Venus } & $\mathrm{K}$ & 5.8 & 3.3 & 0.0 & 3.3 & - & - \\
\hline & & $\mathrm{G}$ & 3.0 & 1.6 & 0.0 & 1.7 & - & - \\
\hline & & $\mathrm{F}$ & 1.9 & 1.0 & 0.0 & 1.0 & - & - \\
\hline \multirow{3}{*}{$\mathrm{C} 2$} & small & $\mathrm{K}$ & 47 & 46 & 46 & 17 & - & - \\
\hline & ocean- & $\mathrm{G}$ & 26 & 25 & 25 & 8.6 & - & - \\
\hline & planet & $\mathrm{F}$ & 17 & 16 & 16 & 5.2 & - & - \\
\hline \multirow{3}{*}{ A3 } & \multirow{3}{*}{ super-Earth } & $\mathrm{K}$ & 4.6 & 1.1 & 0.9 & 0.5 & - & 0.1 \\
\hline & & G & 2.5 & 0.6 & 0.4 & 0.2 & - & 0.1 \\
\hline & & $\mathrm{F}$ & 1.7 & 0.4 & 0.3 & 0.1 & - & 0.0 \\
\hline \multirow{3}{*}{ B3 } & \multirow{3}{*}{ super-Venus } & $\mathrm{K}$ & 5.6 & 0 & 0 & 0 & - & - \\
\hline & & $\mathrm{G}$ & 2.9 & 0 & 0 & 0 & - & - \\
\hline & & $\mathrm{F}$ & 1.9 & 0 & 0 & 0 & - & - \\
\hline \multirow{3}{*}{ C3 } & big & $\mathrm{K}$ & 20 & 13 & 12 & 3.2 & - & - \\
\hline & ocean- & G & 10 & 6.5 & 6.3 & 1.5 & - & - \\
\hline & planet & $\mathrm{F}$ & 6.7 & 4.1 & 4.0 & 0.9 & - & - \\
\hline
\end{tabular}

study: its bands at $620,700,760$, and $1260 \mathrm{~nm}$ are too thin to appear with only $10 \mathrm{~nm}$ resolution. Besides, its Rayleighscattering cross section almost completely masks its absorption features (see Fig. 5), so that no large bands of $\mathrm{O}_{2}$ can be used as an indicator of its presence. However, note that the presence of $\mathrm{O}_{3}$ indirectly indicates the presence of $\mathrm{O}_{2}$, as pointed out by Léger et al. (1993) and others.

\subsubsection{Rayleigh scattering}

When Hartley and Chappuis bands of $\mathrm{O}_{3}$ are absent (all cases but A1), the Rayleigh-scattering signature is clearly visible in the blue part of the spectrum ratio. On one side, it masks the presence of some transitions, like those of $\mathrm{O}_{2}$ and some of $\mathrm{CO}_{2}$, but on the other it can provide two important pieces of information: (i) even if the spectral features cannot be distinguished because they are too thin or faint, the characteristic rising "continuum" as $\lambda^{-4}$ for short wavelengths is a clear indication that the planet has an atmosphere, and (ii) it indirectly indicates the presence of the most abundant species of the atmosphere, such as $\mathrm{CO}_{2}$ and $\mathrm{N}_{2}$, even if $\mathrm{N}_{2}$ shows no spectral signature in the observed domain. As a consequence, Rayleigh scattering can be considered a way to detect $\mathrm{N}_{2}$, provided clouds and/or aerosols do not in turn mask the Rayleigh-scattering signature.
To summarize, it is possible to detect the atmosphere of a transiting exoplanet thanks to the Rayleigh scattering, whatever the composition of the atmosphere. Moreover, it is theoretically possible to distinguish between an $\mathrm{O}_{2}$-rich atmosphere, where $\mathrm{O}_{3}$ is expected to be present (Léger et al. 1993; Sagan et al. 1993), and an $\mathrm{H}_{2} \mathrm{O}$-rich atmosphere, as the $\mathrm{O}_{3}$ lifetime is supposed to be extremely brief when in a water-rich environment. In other words, we should be able to distinguish telluric Earth-like planets with low volatile content from volatile-rich planets. On the other hand, high spectral resolution is needed to distinguish between $\mathrm{H}_{2} \mathrm{O}$-rich planets and Cytherean worlds (B1, B2, B3).

\subsection{Parameters influencing the signal-to-noise ratio}

\subsubsection{Influence of the star}

From Table 3 it is clear that the best targets are K-type stars, rather than G- or F-type stars, the first allowing much better $S / N$ than the latter. Two factors determine the role of the star in whether an exoplanet atmosphere is detected: (i) the size $R_{\star}$ of the star, which directly influences both the $S / N$ (see Eq. (11)) and the duration of transit (Eq. (10)), and (ii) the semi-major axis of the planet's orbit, which influences both the duration of transit and the probability of observing the transit from Earth 
Table 4. Summary of results: mirror effective size and number of targets. (a) Effective size $(\epsilon D)_{S / N \geq 5, V=8}$ of the telescope mirror required to obtain $S / N=5$ for a $V=8$ star, based on the numbers displayed for the models with clouds (see Table 3). (b) The limiting magnitude at which the number of targets in the last column is given. This can be expressed as $\left(V_{\mathrm{Lim}}\right)_{S / N \geq 5, \epsilon D=10 \mathrm{~m}}=5 \cdot \log _{10}\left[\left(S / N_{V=8, \epsilon D=10}\right) / 5 \cdot(\epsilon D) / 10 \mathrm{~m}\right]+8$. (c) Total number of given spectral-type stars brighter than the limiting magnitude. (d) Number of potential targets calculated with Eq. (15), using the $S / N$ value of the models with clouds and assuming various $\beta \cdot \gamma$ values. The coefficients $\beta$ and $\gamma$ are defined in the text. When the number of potential targets is slightly less than 1, the value is given between parenthesis. Use Eq. (16) to scale the value displayed in the column to any mirror effective size $\epsilon D$ and minimum $S / N$.

\begin{tabular}{|c|c|c|c|c|c|c|c|c|}
\hline \multirow[t]{2}{*}{ Model } & \multirow[t]{2}{*}{ Description } & \multirow[t]{3}{*}{ Star } & \multirow{2}{*}{$\begin{array}{c}\text { Mirror } \\
\text { eff. size }(\mathrm{m}) \\
(\epsilon D)_{S / N \geq 5, V=8} \\
\text { W/ clouds }\end{array}$} & \multirow{2}{*}{$\begin{array}{c}\text { Limiting } \\
\text { magnitude } \\
\left(V_{\mathrm{Lim}}\right)_{S / N \geq 5, \epsilon D=10 \mathrm{~m}} \\
\text { W/ clouds }\end{array}$} & \multirow[t]{2}{*}{$\begin{array}{c}\text { Number } \\
\text { of stars }\end{array}$} & \multicolumn{3}{|c|}{$\begin{array}{c}\text { Number of targets } \\
\text { for models w/ clouds } \\
(N)_{S / N \geq 5}, \epsilon=50 \%\end{array}$} \\
\hline & & & & & & $\begin{array}{l}\beta \cdot \gamma=1 \\
D=20 \mathrm{~m}\end{array}$ & $\begin{array}{c}\beta \cdot \gamma=3 \% \\
D=30 \mathrm{~m}\end{array}$ & $\begin{array}{c}\beta \cdot \gamma=10 \% \\
D=30 \mathrm{~m}\end{array}$ \\
\hline & & & (a) & (b) & (c) & & (d) & \\
\hline \multirow{3}{*}{ A1 } & \multirow{3}{*}{$(\approx)$ Earth } & $\mathrm{K}$ & 14 & 7.22 & 2042 & 14 & 1 & 4 \\
\hline & & $\mathrm{G}$ & 22 & 6.31 & 96 & $<1(0.4)$ & $\ll 1$ & $<1(0.1)$ \\
\hline & & $\mathrm{F}$ & 29 & 5.66 & 118 & $<1(0.3)$ & $\ll 1$ & $<1(0.1)$ \\
\hline \multirow{3}{*}{ B1 } & \multirow{3}{*}{$(\approx)$ Venus } & $\mathrm{K}$ & 21 & 6.31 & 580 & 4 & $<1(0.4)$ & 1 \\
\hline & & $\mathrm{G}$ & 43 & 4.90 & 13 & $\ll 1$ & $\ll 1$ & $\ll 1$ \\
\hline & & $\mathrm{F}$ & 68 & 3.73 & 8 & $\ll 1$ & $\ll 1$ & $\ll 1$ \\
\hline \multirow{3}{*}{$\mathrm{C} 1$} & medium & $\mathrm{K}$ & 1.3 & 12.5 & $>3 \times 10^{6}$ & 19602 & 1984 & 6615 \\
\hline & ocean- & $\mathrm{G}$ & 2.5 & 11.0 & 63095 & 321 & 32 & 108 \\
\hline & planet & $\mathrm{F}$ & 3.9 & 10.1 & 54591 & 157 & 15 & 52 \\
\hline \multirow{3}{*}{$\mathrm{A} 2$} & \multirow{3}{*}{ small Earth } & $\mathrm{K}$ & 8 & 8.50 & 11971 & 84 & 8 & 28 \\
\hline & & $\mathrm{G}$ & 13 & 7.51 & 508 & 2 & $<1(0.2)$ & $<1(0.6)$ \\
\hline & & $\mathrm{F}$ & 17 & 6.90 & 656 & 1 & $<1(0.1)$ & $<1(0.3)$ \\
\hline \multirow{3}{*}{ B2 } & \multirow{3}{*}{ small Venus } & $\mathrm{K}$ & 15 & 7.10 & 1730 & 12 & 1 & 4 \\
\hline & & G & 31 & 5.52 & 32 & $<1(0.1)$ & $\ll 1$ & $\ll 1$ \\
\hline & & $\mathrm{F}$ & 50 & 4.50 & 23 & $\ll 1$ & $\ll 1$ & $\ll 1$ \\
\hline \multirow{3}{*}{$\mathrm{C} 2$} & small & $\mathrm{K}$ & 1.1 & 12.8 & $>4 \times 10^{6}$ & 33569 & 3398 & 11329 \\
\hline & ocean- & $\mathrm{G}$ & 2.0 & 11.5 & 125892 & 600 & 60 & 202 \\
\hline & planet & $\mathrm{F}$ & 3.1 & 10.5 & 94868 & 307 & 31 & 103 \\
\hline \multirow{3}{*}{$\mathrm{A} 3$} & \multirow{3}{*}{ super-Earth } & $\mathrm{K}$ & 45 & 4.71 & 63 & $<1(0.4)$ & $\ll 1$ & $<1(0.1)$ \\
\hline & & $\mathrm{G}$ & 86 & 3.39 & 1 & $\ll 1$ & $\ll 1$ & $\ll 1$ \\
\hline & & $\mathrm{F}$ & 121 & 2.51 & 1 & $\ll 1$ & $\ll 1$ & $\ll 1$ \\
\hline \multirow{3}{*}{ B3 } & \multirow{3}{*}{ super-Venus } & $\mathrm{K}$ & $>10^{3}$ & - & 0 & $\ll 1$ & $\ll 1$ & $\ll 1$ \\
\hline & & $\mathrm{G}$ & $>10^{3}$ & - & 0 & $\ll 1$ & $\ll 1$ & $\ll 1$ \\
\hline & & $\mathrm{F}$ & $>10^{3}$ & - & 0 & $\ll 1$ & $\ll 1$ & $\ll 1$ \\
\hline \multirow{3}{*}{$\mathrm{C} 3$} & big & $\mathrm{K}$ & 4.0 & 10.1 & 109182 & 682 & 69 & 230 \\
\hline & ocean- & $\mathrm{G}$ & 7.7 & 8.57 & 2197 & 10 & 1 & 3 \\
\hline & planet & $\mathrm{F}$ & 13 & 7.57 & 1656 & 4 & $<1(0.4)$ & 1 \\
\hline
\end{tabular}

(see below). These factors can explain the discrepancies between the $S / N$ values obtained for different kinds of stars in Table 3.

The probability, $\alpha$, that a planet transiting its parent star might be seen from the Earth is defined as $\alpha \equiv P\{$ transit $\}=$ $R_{\star} / a$, with $R_{\star}$ the radius of the star and $a$ the semi-major axis of the planet's orbit. This probability is about $10 \%$ for "hot Jupiters", while it is $0.3 \%, 0.5 \%$, and $0.7 \%$ for planets orbiting in the $\mathrm{HZ}$ of a F, G, or $\mathrm{K}$ star, respectively.

In addition, $\mathrm{K}$ stars are more numerous than other types of stars. From the CDS database, we find there is approximatively a total of $10000 \times 10^{0.6(V-8)}$ main sequence stars brighter than a given magnitude $V$ on the whole $\mathrm{sky}^{8}$. About $3 / 5$ of these are $\mathrm{K}$ type stars, against only $1 / 10$ for $\mathrm{G}$ stars. Let us now define $\beta$

${ }_{8}$ We consider mostly bright stars, for which the distribution is essentially isotropic. to be the number of planet(s) per star, and $\gamma$ to be the fraction of the sky that is considered for a transit detection survey (in other words the efficiency of surveys to find the targets). We list in Table 4 the number of potential targets for each model. This number, $N$, corresponds to the number of targets detected with a 10-m telescope mirror effective size and with an $S / N$ greater than or equal to 5 . This is given by

$N_{S / N \geq 5, \epsilon D=10 \mathrm{~m}}=N_{0} \cdot \alpha \cdot \beta \cdot \gamma \cdot\left(\frac{S / N_{V=8, \epsilon D=10 \mathrm{~m}}}{5}\right)^{3}$,

where $N_{0}$ is about 6000,1000 , and 3000 for $\mathrm{K}, \mathrm{G}$, and F stars respectively; i.e. the number of stars with magnitude $\geq 8$, and $S / N_{V=8, \epsilon D=10 \mathrm{~m}}$ is the expected $S / N$ ratio computed for a given atmosphere of a planet orbiting a $V=8$ star with a $10-\mathrm{m}$ mirror effective size (this value is given in the last column of Table 3). Since no Earth-size planet has been discovered so far, 

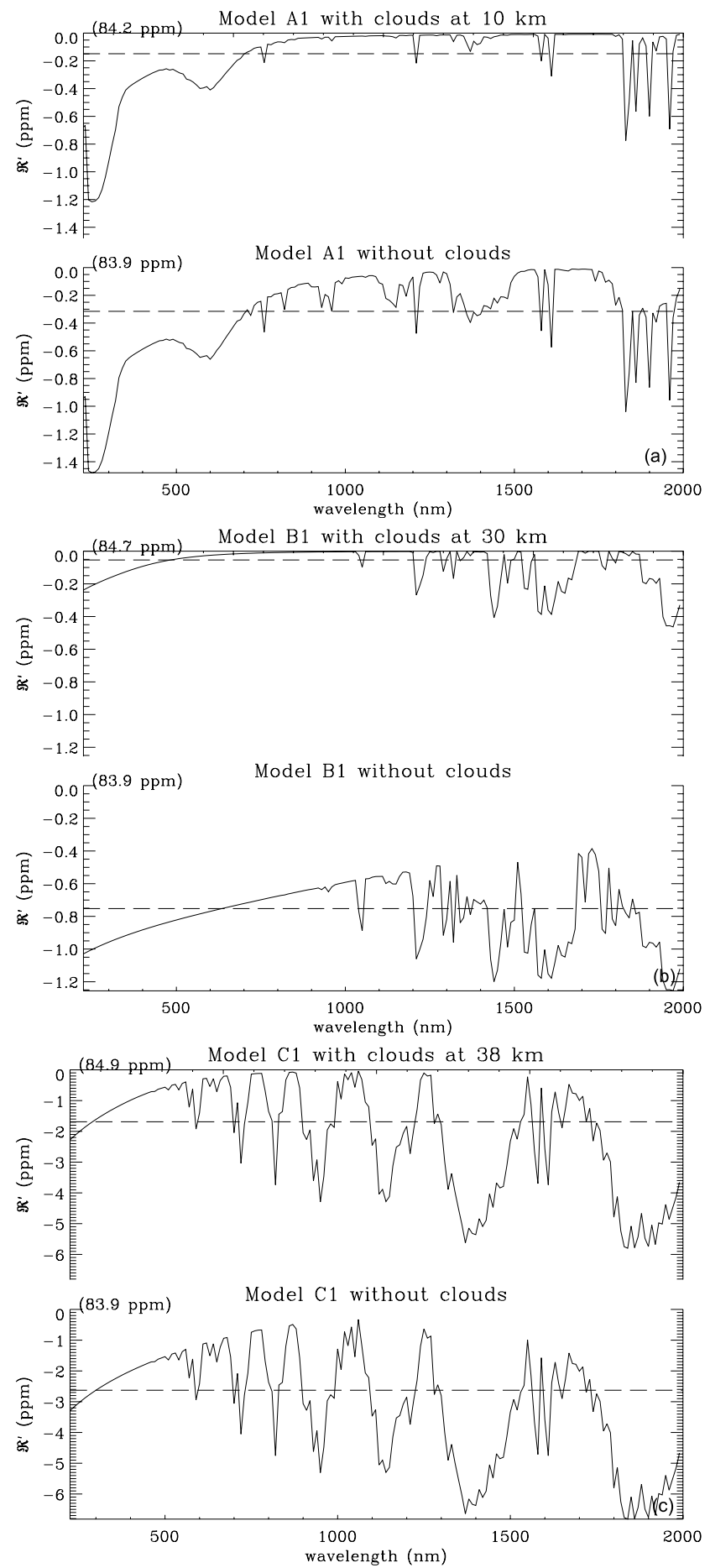

Fig. 8. Spectrum ratios for models A1 a), B1 b), and C1 c). The spectrum ratios have been respectively shifted by the values in parenthesis so that the absorption by the "solid disk" of the planet is $0 \mathrm{ppm}$. In the case of models with clouds, the "solid disk" is artificially increased by the cloud layer. The dashed line indicates the best-fit estimation of the radius of the planet, $\tilde{R}_{\mathrm{P}}$ (see Sect. 3 ), if we suppose there is no atmosphere.

we have no real estimate for $\beta$. In the following, when it is not a free parameter, we consider that $\beta=1^{9}$. Catala et al. (2005)

\footnotetext{
9 Actually, $\beta=2$ in the Solar System, because there are two Earthsize planets with atmospheres, namely Venus and the Earth.
}
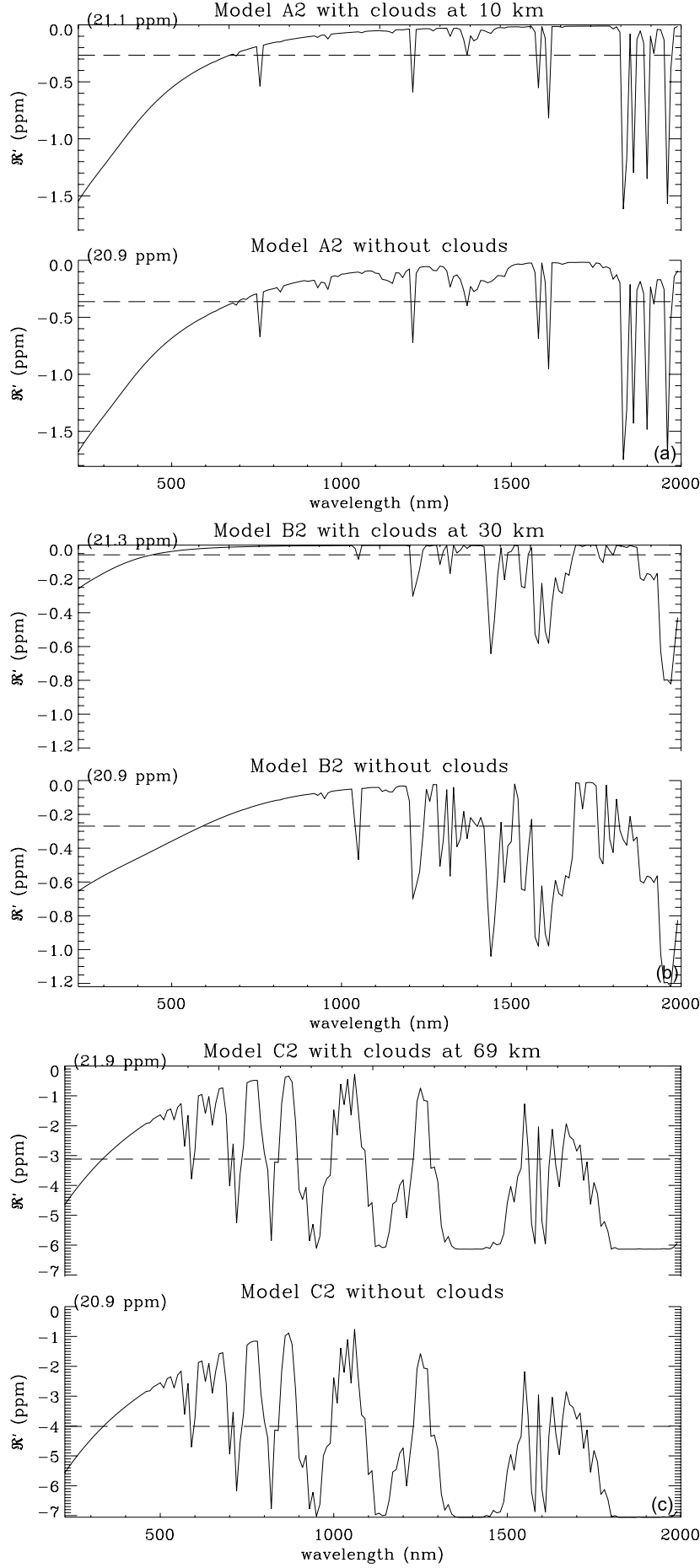

Fig. 9. Spectrum ratios for models $\mathrm{A} 2$ a), B2 b), and $\mathrm{C} 2$ c). The "saturation effect" in $\mathrm{H}_{2} \mathrm{O}$ lines for model $\mathrm{C} 2$ is a consequence of the atmosphere being optically thick at the upper atmospheric level, $h_{\max }$. In fact, if one considers there is no more water above this level due to photo-dissociation (see Sect. 2.2.3), such transmitted spectrum plots allow the level where $\mathrm{H}_{2} \mathrm{O}$ photo-dissociation occurs in an exoplanet atmosphere to be determined.

propose a $30^{\circ} \times 30^{\circ}$ survey dedicated to finding planets around $<11$ th-magnitude stars, i.e., $\gamma \approx 2-3 \%$ for such a project.

Let $N_{S / N, \epsilon D}$ be the number of potential targets reaching a minimum $S / N$ ratio for a given mirror effective size $\epsilon D$, which 

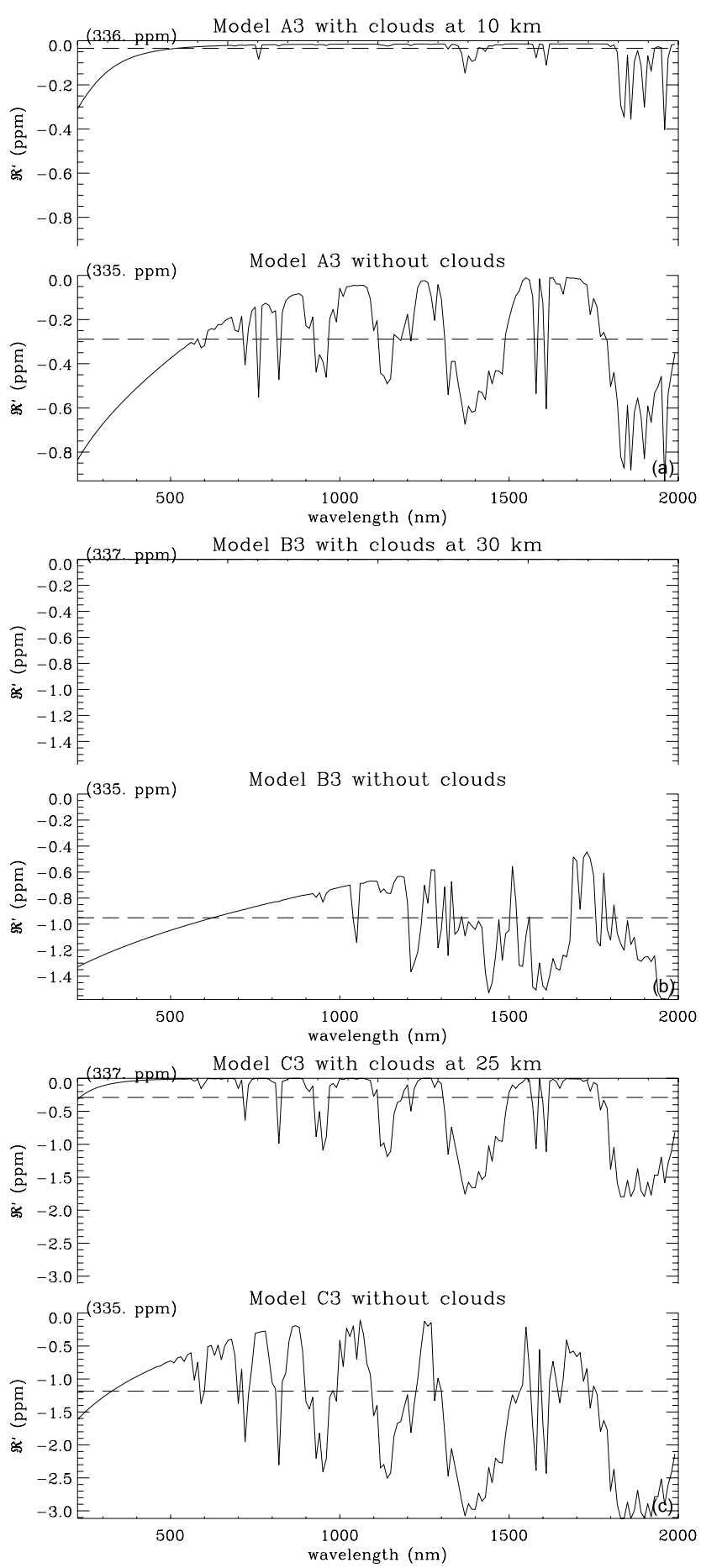

Fig. 10. Spectrum ratios for models $A 3$ a), B3 b), and C3 c).

scales from the value calculated using Eq. (15), $N_{S / N \geq 5, \epsilon D=10 \mathrm{~m}}$, in the following way:

$N_{S / N, \epsilon D}=N_{S / N \geq 5, \epsilon D=10 \mathrm{~m}} \cdot\left(\frac{S / N}{5}\right)^{-3} \cdot\left(\frac{\epsilon D}{10 \mathrm{~m}}\right)^{3}$.

The values obtained for atmospheric detection are strongly in favor of a small, late-type star. Note that this is also true for the detection of the planetary transit as well.

\subsubsection{Effect of the atmospheric temperature gradient}

The thick $\mathrm{CO}_{2}$ Venus-like atmospheres (B1, B2, and B3, see Tables 3 and 4) are more difficult to detect than other cases. Even if we set the top of the clouds at a $10-\mathrm{km}$ height, the detection remains more challenging than for model $\mathrm{A} 1$. That is somewhat surprising partly because $\mathrm{CO}_{2}$ has strong transitions, particularly in the near infrared, and partly because of the larger scale height at the surface of the planet $(14.3 \mathrm{~km}$ for model B1, $8.8 \mathrm{~km}$ for model A1). As a consequence, the atmosphere in model B1 should have a larger vertical extent than in model A1. In reality, it is difficult to detect the atmospheres of models B1, B2, and B3 because of their temperature profiles (see Figs. 2 and 3): at $50 \mathrm{~km}$ of altitude, the temperature of model B1 is roughly $60 \mathrm{~K}$ colder than model A1. This model in fact benefits from the positive stratospheric temperature gradient of the Earth. Moreover, the atmosphere for model B1 $\left(\mu_{\mathrm{B} 1}=43 \mathrm{~g} \mathrm{~mol}^{-1}\right)$ is heavier than the one for model A1 $\left(\mu_{\mathrm{A} 1}=29 \mathrm{~g} \mathrm{~mol}^{-1}\right)$. Therefore, at high altitude, the scale height is larger in model A1 than in model B1 $(7.6 \mathrm{~km}$ and $3.9 \mathrm{~km}$, respectively, at an altitude of $50 \mathrm{~km}$ ).

\subsubsection{Effect of atmospheric pressure}

Note that the thickness of the atmosphere in model B1 is almost half the one in A1, despite the intense surface pressure (100 atm), which should help to increase the upper level of the atmosphere, limited by the UV photo-dissociation $\left(h_{\max }\right)$. The exponential decrease in pressure prevents, in fact, $p_{0}$ playing a key role: in order to counterbalance the effect of the negative temperature gradient, the surface pressure should have been $>10^{6}$ atm to obtain absorptions similar to the situation on Earth (model A1).

\subsubsection{Effect of the planet's gravity and density}

The atmospheric absorption is approximately proportional to $H \cdot R_{\mathrm{P}}$. At a given temperature and for a given atmospheric composition, the scale height $H$ is proportional to the inverse of the gravity acceleration, $g^{-1}$, or equivalently to $R_{\mathrm{P}}^{2} / M_{\mathrm{P}}$, where $M_{\mathrm{P}}$ is the mass of the planet. As a result, the absorption is expected to be about inversely proportional to the bulk density of the planet $\rho_{\mathrm{P}}$ and independent of the planet size.

This effect is illustrated by the following examples: models $\mathrm{C} 1, \mathrm{C} 2$, and $\mathrm{C} 3$ all benefit from very extended atmospheres, given the weak value of $g$ in the three cases. For a planet as dense as the Earth (such that $g_{\mathrm{C} 1}=g_{\mathrm{A} 1}$ ), the results for the $\mathrm{N}_{2} / \mathrm{H}_{2} \mathrm{O}$-rich atmosphere in models $\mathrm{C}$ are close to the ones obtained for the A models. Both models $\mathrm{C}$ and A present typical spectral features. In model A1, ozone, for which the concentration peaks at the tropopause, gives a prominent signature in the blue edge of the spectral domain (the Hartley and Chappuis bands, as seen in Fig. 8, top panel). In contrast, the saturated atmosphere of model $\mathrm{C} 1$, which sustains $\mathrm{H}_{2} \mathrm{O}$ up to high altitudes, yields strong bands around 0.14 and $0.19 \mu \mathrm{m}$ (Fig. 8, bottom panel). The role played by $g$ can be better understood by comparing model A3 or B3 $\left(g=24.5 \mathrm{~m} \mathrm{~s}^{-2}\right)$ to model A2 or B2 $\left(g=3.9 \mathrm{~m} \mathrm{~s}^{-2}\right)$, and model C3 $\left(g=14.7 \mathrm{~m} \mathrm{~s}^{-2}\right)$ to model C2 
$\left(g=2 \mathrm{~m} \mathrm{~s}^{-2}\right)$. Using absorption spectroscopy, it is clear that the atmospheres of small and light planets (i.e., with low surface gravity) are easier to detect than those of large and dense planets (i.e., with high surface gravity).

Small and light exoplanets, however, may not be able to retain a thick atmosphere. In fact, high thermal agitation of atmospheric atoms causes particles to have a velocity in the tail of the Maxwellian distribution that allows them to escape into space (i.e., Jean's escape). It is therefore questionable whether planets the size of Titan can have a dense atmosphere at $1 \mathrm{AU}$ from their star. Models A2, B2, and C2 join that category. This problem concerns both small planets and giant exoplanet satellites.

According to Williams et al. (1997), a planet with the density of Mars could retain $\mathrm{N}$ and $\mathrm{O}$ over more than $4.5 \mathrm{Gyr}$ if it has a mass greater than $0.07 M_{\oplus}$. Model planets A2 and B2 have masses of $0.1 M_{\oplus}$ and a density equivalent to that of Mars $\left(\approx 4 \mathrm{~g} \mathrm{~cm}^{-3}\right)$, so they would be able to retain an atmosphere (though they may not be able to have a $1 \mathrm{~atm}$ atmosphere, as for Mars). The ocean-planet model C2 has a mass of $0.05 M_{\oplus}$ for a density of $2.8 \mathrm{~g} \mathrm{~cm}^{-3}$, and according to Williams et al. (1997), its atmosphere should consequently escape. However, although 1 AU from the star, such a planet also has a huge reservoir of volatile elements. This reservoir should help to "refill" the escaping atmosphere.

Note that a hydrodynamically escaping atmosphere should be easier to detect than a stable one, since it can bring heavier elements into the hot upper atmosphere. This effect is illustrated by the absorptions seen by Vidal-Madjar et al. (2003, 2004) in the spectrum of HD 209458, which originate in its transiting giant-planet hydrodynamically escaping atmosphere. A model of an "escaping ocean" is studied by Jura (2004). This process would give interesting absorption signatures in the $\mathrm{H}_{2} \mathrm{O}$ bands from the lower atmosphere and in the signatures of the photo-dissociation products of $\mathrm{H}_{2} \mathrm{O}$ from the upper atmosphere (such as an absorption of $\mathrm{Ly}_{\alpha}$ photons by the hydrogen atom); see the detailed discussion in Jura (2004).

\section{Conclusion}

The vertical extent of the atmosphere is of extremely important for detecting a remote atmosphere by absorption spectroscopy. This tends to favor less dense objects, like giant exoplanet satellites, such as an "exo-Titan", or volatile-rich planets, such as ocean-planets, theoretically possible but not observed yet. Cytherean atmospheres are the most challenging to detect. Surface parameters, such as surface pressure and temperature, are not crucial. A temperature gradient that becomes positive at a few tens of kilometers height (for instance owing to photochemistry) might help the detection. Our results show that late-type stars are better for detecting and characterizing the atmospheres of planets in transit, since they are smaller, more numerous, and they present a better probability of being transited by a planet.

The strongest signatures of the atmosphere of a transiting Earth-size planet could be those of $\mathrm{H}_{2} \mathrm{O}(6 \mathrm{ppm}$ in the case of hypothetical ocean-planets), $\mathrm{O}_{3}(\sim 1-2 \mathrm{ppm})$, and $\mathrm{CO}_{2}$ (1 ppm), considering our spectral study from the UV to the NIR (i.e., from 0.2 to $2 \mu \mathrm{m}$ ). The presence of an atmosphere around hundreds of hypothetical "ocean-planets" (models C) could be detected with a 10-20 m telescope. The atmospheres of tens of giant exoplanet satellites (model A2) could be in the range of a 20-30 m instrument. A 30-40 m telescope would be required to probe Earth-like atmospheres around Earth-like planets (model A1). These numbers suppose that Earth-size planets are frequent and are efficiently detected by surveys.

Finally, planets with an extended upper atmosphere, like the ones described by Jura (2004), hosting an "evaporating ocean", or the planets in an "hydrodynamical blow-off state" are the natural links between the planets we have modelled here and the observed "hot Jupiters".

Acknowledgements. We warmly thank Chris Parkinson for careful reading and comments that noticeably improved the manuscript, David Crisp for the code LBLABC, and the anonymous referee for a thorough reading and useful comments on the manuscript. This work is supported by CNRS - INSU Programme National de Planétologie and Groupement de Recherche en Exobiologie. G. Tinetti is supported by NASA Astrobiology Institute - National Research Council.

\section{References}

Aigrain, S., Favata, F., \& Gilmore, G. 2004, A\&A, 414, 1139

Bates, D. R. 1984, P\&SS, 32, 785

Brown, T. M. 2001, ApJ, 553, 1006

Catala, C., Aerts, C., Aigrain, S., et al. 2005, Proc. 39th ESLAB Symposium, Noordwijk, 19-21 April 2005, ed. F. Favata, \& A. Gimenez

Charbonneau, D., Brown, T. M., Latham, D. W., et al. 2000, ApJ, 529, 45

Charbonnneau, D., Brown, T. M., Noyes, R. W., \& Gilliland, R. L. 2002, ApJ, 568, 377

Dubois, V. 2002, Ph.D. Thesis, Université de Nantes - Observatoire de Paris

Elliot, J. L., \& Olkin, C. B. 1996, ARE\&PS, 24, 89

Ehrenreich, D., Despois, D., Léger, A., et al. 2005, in preparation

Fishbein, E., Farmer, C. B., Granger, S. L., et al. 2003, IEEE Transactions on Geoscience and Remote Sensing, 41, 2

Green, D., Matthews, J., Seager, S., \& Kuschnig, R. 2003, ApJ, 597, 590

Hubbard, W. B., Fortney, J. J., Lunine, J. I., et al. 2001, ApJ, 560, 413 Jacquinet-Husson, N., Arié, E., Ballard, J., et al. 1999, JQSRT, 62, 205 Jura, M. 2004, ApJ, 605, 65

Kasting, J. F., Whitmire, D. P., \& Reynolds, R. T. 1993, Icarus, 101, 108

Kliore, A. J., Moroz, V. I., \& Keating, G. M. 1985, ed., Adv. Space Res., 5, 11, 1

Henry, G. W., Marcy, G. W., Butler, R. P., et al. 2000, ApJ, 529, 41

Lanza, A. F., Rodonò, M., \& Pagano, I. 2004, A\&A, 425, 707

Lecavelier des Etangs, A., Vidal-Madjar, A., McConnell, J. C., \& Hébrard, G. 2004, A\&A, 418, 1

Léger, A., Pirre, M., \& Marceau, F. J. 1993, A\&A, 277, 309

Léger, A., Selsis, F., Sotin, C., et al. 2004, Icarus, 169, 499

Mazeh, T., Naef, D., Torres, G., et al. 2000, ApJ, 532, 55

McArthur, B. E., Endl, M., Cochran, W. D., et al. 2004, ApJ, 614, 81

Meadows, V. S., \& Crisp, D. 1996, JGR, 101, 4595

Moutou, C., Pont, F., Barge, P., et al. 2005, A\&A, 437, 355

Naus, H., \& Ubachs, W. 2000, Optics Letters, 25, 347

Rivera, E., Lissauer, J., Butler, P., et al. 2005, ApJL, submitted 
Rothman, L. S., Barbe, A., Benner, D. C., et al. 2003, JQSRT, 82, 5 Sagan, C., Thomson, W. R., Carlson, R., et al. 1993, Nature, 365, 715 Santos, N. C., Bouchy, F., Mayor, M., et al. 2004, A\&A, 426, 19

Sato, B., Fischer, D. A., Henry, G. W., et al. 2005, ApJ, 633, 465

Schiebener, P., Straub, J., Levelt Sengers, J. M. H., et al. 1990, J. Phys. Chem. Ref. Data, 19, 677

Schneider, J. 1994, Astr. \& Spa. Sci., 212, 321

Seager, S., \& Sasselov, D. 2000, ApJ, 537, 916

Segura, A., Krelove, K., Kasting, J. F., et al. 2003, Astrobiology, 3, 689

Sneep, M., \& Ubachs, W. 2005, JQSRT, 92, 293

Sotin, C., et al. 2005, A\&A, submitted

Tian, F., Toon, O. B., Pavlov, A. A., et al. 2005, ApJ, 621, 1049

Tinetti, G., Meadows, V. S., Crisp, D., et al. 2005, Astrobiology, 5, 461
Tinetti, G., Meadows, V. S., Crisp, D., et al. 2005, Astrobiology, submitted [arXiv: astro-ph/0502238]

Triplet, J.-P., \& Roche, G. 1986, Météorologie générale, ed. Météo France, 3rd edition

Vidal-Madjar, A., Lecavelier des Etangs, A., Désert, J.-M., et al. 2003, Nature, 422, 143

Vidal-Madjar, A., Désert, J.-M., Lecavelier des Etangs, A., et al. 2004, ApJ, 604, 69

Webb, J. K., \& Wormleaton, I. 2001, Publ. Astron. Soc. Aust., 18, 252 Williams, D. M., Kasting, J. F., \& Wade, R. A. 1997, Nature, 385, 234 Wolszczan, A., \& Frail, D. A. 1992, Nature, 355, 145

Zombeck, M. V. 1990, Handbook of space astronomy \& astrophysics (Cambridge University Press), 2nd edition 